Annales Geophysicae (2003) 21: 847-862 (c) European Geosciences Union 2003

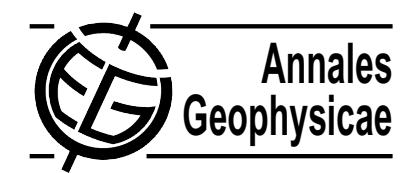

\title{
On the solar origin of interplanetary disturbances observed in the vicinity of the Earth
}

\author{
N. Vilmer ${ }^{1}$, M. Pick ${ }^{1}$, R. Schwenn ${ }^{2}$, P. Ballatore* ${ }^{*}$ and J. P. Villain ${ }^{3}$ \\ ${ }^{1}$ LESIA - UMR CNRS 8109 - Paris Observatory, 5 Place J. Janssen, F-92195 Meudon Cedex, France \\ ${ }^{2}$ Max Planck Institut für Aeronomie, Max-Planck-Straße 2, D-37191 Katlenburg-Lindau, Germany \\ ${ }^{3}$ LPCE/CNRS Orléans, 3A av. de la Recherche Scientifique, F-45071 Orléans, France \\ * on leave from LPCE/CNRS Orléans, 3A av. de la Recherche Scientifique, F-45071 Orléans, France
}

Received: 13 May 2002 - Revised: 5 September 2002 - Accepted: 1 October 2002

\begin{abstract}
The solar origin of 40 interplanetary disturbances observed in the vicinity of the Earth between January 1997 and June 1998 is investigated in this paper. Analysis starts with the establishment of a list of Interplanetary Mass Ejections or ICMEs (magnetic clouds, flux ropes and ejecta) and of Interplanetary Shocks measured at WIND for the period for which we had previously investigated the coupling of the interplanetary medium with the terrestrial ionospheric response. A search for associated coronal mass ejections (CMEs) observed by LASCO/SOHO is then performed, starting from an estimation of the transit time of the interplanetary perturbation from the Sun to the Earth, assumed to be achieved at a constant speed (i.e. the speed measured at 1 $\mathrm{AU})$. EIT/SOHO and Nançay Radioheliograph (NRH) observations are also used as proxies in this identification for the cases when LASCO observations do not allow one to firmly establish the association. The last part of the analysis concerns the identification of the solar source of the CMEs, performed using a large set of solar observations from X-ray to radio wavelengths. In the present study, this association is based on a careful examination of many data sets (EIT, NRH and $\mathrm{H} \alpha$ images and not on the use of catalogs and of Solar Geophysical Data reports). An association between interplanetary disturbances and LASCO/CMEs or proxies on the disk is found for 36 interplanetary events. For 32 events, the solar source of activity can also be identified. A large proportion of cases is found to be associated with a flare signature in an active region, not excluding of course the involvement of a filament. Conclusions are finally drawn on the propagation of the disturbances in the interplanetary medium, the preferential association of disturbances detected close to the Earth's orbit with halos or wide CMEs and the location on the solar disk of solar sources of the interplanetary disturbances during that period.
\end{abstract}

Correspondence to: N. Vilmer (nicole.vilmer@obspm.fr)
Key words. Interplanetary physics (interplanetary shocks); solar physics, astrophysics and astronomy (flares and mass ejections)

\section{Introduction}

The relationship between the large-scale structures of plasmas and magnetic fields expelled from the Sun (Coronal Mass Ejections or CMEs) and transient interplanetary disturbances, such as shocks and magnetic clouds or flux ropes, has been studied extensively, using, for example, Solwind and Helios 1 observations around 1980 (e.g. Sheeley et al., 1985 ) and for the present solar cycle SOHO and WIND observations (e.g. Webb et al., 2000; Gopalswamy et al., 2000a, 2001a; St Cyr et al., 2000; Cane et al., 2000). In particular, a lot of attention has been devoted recently to the observations of Halo CMEs, i.e. expanding halo-like brightenings surrounding the occulter of the coronagraph and moving outwards along the Sun-Earth line. Indeed, after a transit time of two to five days in the interplanetary medium, these disturbances can potentially reach the Earth's orbit and produce transient events measured in situ at $1 \mathrm{AU}$ (e.g. Gosling, 1996).

Interplanetary shocks (e.g. observed by the Helios solar probes) are known to be associated with large, low-latitude coronal mass ejections on the nearby limb (e.g. Schwenn, 1983; Sheeley et al., 1985). Magnetic clouds (MC) in the interplanetary medium (see below for a precise definition) have been often associated with $\mathrm{H} \alpha$ eruptive prominences or disappearing filaments at the Sun (DSF) (i.e. Burlaga et al., 1982; Wilson et al., 1986; Bothmer and Schwenn, 1994). More recently, Bothmer and Rust (1997) confirmed this association for magnetic clouds observed by spacecraft near the Earth, using the measured plasma speed of the cloud to 
estimate the onset time of the event at the Sun. Although a convincing association between magnetic clouds and disappearing filaments was found, several difficulties were emphasized:

1. It is always difficult to make a unique association between interplanetary and solar events;

2. For a large number of events, the associated filament disappearance was observed quite far away from the Sun's central meridian;

\section{Multiple DSFs were observed for some cases.}

Most of the studies on the association between magnetic clouds and solar sources of activity were, however, primarily focussed on the search of $\mathrm{H} \alpha$ filaments or prominences, not investigating other sources of solar activity, such as flares (see below).

Many studies have also investigated the solar source of CMEs and the link between CMEs and solar active phenomena. It was concluded that CMEs, are associated either with flares or with eruptive prominences or disappearing filaments on the disk (i.e. Sheeley et al., 1975, 1983; Gosling et al., 1974, 1976; Munro et al., 1979; Webb and Hundhausen, 1987; St Cyr and Webb, 1991), with a percentage of association varying among the different studies. Conversely, Gilbert et al. (2000) studied the relationship between prominence activity and CMEs, and found that eruptive protuberances (EP) are more strongly associated with CMEs than active prominences (AP). It was also found that CMEs associated with eruptive prominences show a significant acceleration in the corona, while flare-associated ones most often propagate with a constant speed in the corona (i.e. MacQueen and Fisher, 1983). This result was reconfirmed with more recent observations of CMEs with SOHO/LASCO (Srivastava et al., 2000). Andrews and Howard (2001) also concluded that two types of CMEs can be easily distinguished by examining the height-time plots: type A (for acceleration) events corresponding to a constant acceleration (within 25 solar radii) are usually associated with pre-existing helmetstreamers and often with prominence eruptions or filament disappearances. The type $\mathrm{C}$ (constant speed) events which are usually brighter, larger and faster (in the corona) than type A events may be associated with X-ray flares. More recently, Gopalswamy et al. (2001b) found a new class of CME events (usually associated with long wavelengths radio bursts), exhibiting some deceleration within 25 solar radii. It has been known since Sheeley et al. (1975) that there is a good association between CMEs and soft X-ray flares, with a probability of association increasing with the X-ray flare duration reaching $100 \%$ for X-ray flares lasting longer than six hours (see also Webb, 1992 for a review). St Cyr and Webb (1991) and Harrison (1995) also found a mean association rate of $48 \%$. Considering that roughly half the number of CMEs are initiated from the back side of the Sun and cannot produce detectable X-ray emission from the Earth, Zhang et al. (2001) concluded that it is justified to consider that a large number of CMEs are associated with X-ray flares. Soft X-ray and EUV "dimmings" have also been observed on the solar disk as the direct signature of flares associated with CMEs (Sterling and Hudson, 1997; Thompson et al., 1998, 2000a; Zarro et al., 1999). "EIT waves" observed by the EUV Imaging Telescope (EIT) (Thompson et al., 1999, 2000b) have also been sometimes observed originating from the vicinity of active regions in association with CMEs. The active region may be located far away from the position of the CME observed with the coronagraph, but it is found that the extended dimming areas generally map out the apparent footprints of the CME (Thompson et al., 2000a, b; Pohjolainen et al., 2001). Delannée et al. (2000) also studied the relationship between EUV ejecta observed at $195 \AA$ by EIT and CMEs observed by SOHO/LASCO coronagraphs. They found that among 17 observed ejecta, only 13 gave observable CME signatures in LASCO. They concluded that more CMEs can thus be detected also using EIT observations. Finally, radio emission at metric wavelengths is often observed in association with CMEs. The large-scale radio sources observed at metric wavelengths may be used to trace the ondisk locations of the CME source regions (e.g. Pohjolainen et al., 2001). Faint metric radio loop-like shaped emissions can also be observed in association with white-light CMEs (Maia et al., 2000; Bastian et al., 2001).

The goal of the present study is to perform another investigation on the solar source of interplanetary perturbations observed close to the Earth, using a slightly different approach from the ones followed in previous studies (e.g. Webb et al., 2000; Gopalswamy et al., 2000a, 2001a; St Cyr et al., 2000) in tracing the perturbations of the interplanetary medium back to the Sun. The present analysis starts with the identification of interplanetary perturbations (shocks and magnetic clouds) measured at WIND during the period January 1997June 1998 for which we had investigated in parallel the coupling of the interplanetary medium and of the terrestrial ionospheric response using the observations of the SuperDARN radar network (Ballatore et al., 2001). This period overlaps the period studied in Gopalswamy et al. (2000a, 2001a) and also in St Cyr et al. (2000). However, the present study is not focussed on the most efficient perturbations in terms of geomagnetic activity (i.e. geo-effective events), contrary to the investigations of St Cyr et al. (2000). A search for the associated coronal mass ejections observed by SOHO/LASCO is performed as in Gopalswamy et al. (2000a, 2001a). However, the method used to link interplanetary disturbances and CMEs, as well as the search for the solar source of CMEs are different in the two studies and will be presented in detail in Sect. 2. In this paper, the starting point for the association is the transit time of the perturbation from the Sun to the WIND spacecraft, assumed to be at the constant speed measured at $1 \mathrm{AU}$, as in Lindsay et al. (1999). Gopalswamy et al. (2000a, 2001a) started with a larger search window (1 to 5 days before the interplanetary event). EIT/SOHO and Nançay Radioheliograph (NRH) observations are also used as proxies in the identification of the CME for the cases when LASCO observations by themselves do not allow to firmly establish 
the association. The final results of the present study concern the identification of the solar source of the CMEs. This identification is not based on only the use of catalogues, but is done by analysing a large set of solar observations described in Sect. 2.2. It must finally be noted that the list of CMEs studied in the present paper also overlaps the period of six months of halo CMEs studied in Webb et al. (2000). Our approach is, however, different from the one followed in Webb et al. (2000), since the starting point of our study is a list of interplanetary perturbations, while Webb et al. (2000), starting from the list of halo CMEs, examined WIND data three to five days after, to search for magnetic clouds. The criterium used by Webb et al. (2000) to find the solar sources of activity related to the halo CMEs is also based on the list of solar events published in Solar Geophysical Data (eruptive prominences, $\mathrm{H} \alpha$ flares, SXR events, metric bursts) and on additional information using YOHKOH SXT and EIT data on activity starting $\simeq$ three hours before the leading edge of the CME appears in the field-of-view of $\mathrm{C} 2$ and ending at that time. All the activity (flares, disappearing or eruptive filaments or protuberances) is considered in their study without discriminating between activity associated with active regions or with large-scale filaments, as done in the present study.

\section{Data analysis}

2.1 Association between interplanetary disturbances and coronal mass ejections

The present analysis is performed on a list of interplanetary events from solar origin that we identified using the observations of the Solar Wind and Magnetic Field Instruments aboard the WIND spacecraft (Ogilvie et al., 1995). In this paper, the selected interplanetary events are on one side, Interplanetary Mass Ejections or ICMEs, which are classified in magnetic clouds (MC), flux ropes and ejecta according to the criteria described below, and on the other side, interplanetary shocks, followed or not followed by turbulent plasma sheaths (see below). In agreement with the first definition given in Burlaga et al. (1981), magnetic clouds (MC) are characterized by a smooth and continuous rotation of the magnetic field $B$ following an interplanetary shock. They also correspond to higher values of the strength of the magnetic field, as well as lower values of the temperature (i.e. a small value of $\beta$ ). In a later study based on $46 \mathrm{MC}$, Bothmer and Schwenn (1997) showed that the interplanetary structure characterized by the magnetic field rotation is preceded by a fast forward interplanetary shock in $85 \%$ of the cases. In the following, we shall use the term flux rope for those events for which the continuous rotation of the $B$ field is not preceded by a shock. This is to investigate whether the solar origin may be different in the case where the rotation of the field is preceded or not by a shock. The term ejecta corresponds to a few events where the shock is first followed by a high density structure and then by a well-defined struc- ture with low $\beta$, as for a magnetic cloud, but for which the rotation of the $B$ field is not obvious. We also refer to the discussion and forum on terminology in e.g. Cliver (2001), Burlaga (2001) and Russell (2001). In our classification, the interplanetary shocks may be followed by turbulent plasma sheaths (indicated by shock + sheath), but with no low $\beta$ structure following the shock. Table 1 gives the list of the 40 interplanetary disturbances that were selected during the period January 1997-June 1998. They include 27 ICMEs (21 MC, 2 Ejecta, 4 Flux ropes) and 13 interplanetary shocks. The starting time indicated in Table 1 corresponds for all disturbances, except flux ropes, to the time of the interplanetary shock associated with the feature. For the flux ropes, it is the time when the magnetic field starts to rotate. Except for three cases for which the velocity at WIND reaches 600 to almost $800 \mathrm{~km} / \mathrm{s}$ (9 February 1997, 1 and 4 May 1998), the selected ICMEs have a velocity at WIND not strongly different from the mean speed of the solar wind at $1 \mathrm{AU}$ (i.e. around $450 \mathrm{~km} / \mathrm{s})$. This is in agreement with the former results of Gosling (1997), Lindsay et al. (1999) and Gopalswamy et al. (2000a), who found that most of the ICMEs at 1AU travel with a velocity close to the solar wind speed. Column 8 of Table 1 indicates the values of $K_{p}$ and Dst reported at the time of the arrival of the interplanetary disturbance at Earth.

The following procedure was used to establish the association between ICMEs and interplanetary shocks with CMEs: the inferred onset time (launch window) of the perturbation at the solar surface, $t_{c}$ (column 5 of Table 1), is estimated assuming that the perturbation propagates in the interplanetary medium at a constant velocity, given by the velocity measured at $1 \mathrm{AU}$ by WIND. The uncertainty of $V_{\mathrm{WIND}}$ (column 3 ) is used to determine not only the possible onset time, but also a launch window (column 5). Then, using the list of the CMEs detected by LASCO/SOHO coronagraphs (Brueckner et al., 1995) and identified by St Cyr et al. (2000), up to five CMEs are selected, the closest one in time to $t_{c}$ (column 6 of Table 1 with information on the polar angle (PA) and the width $\left(W_{i}\right)$ of the $\mathrm{CME}$ in $\mathrm{C} 2$; the times indicated in the columns 6 and 7 are the ones of the first appearance in the $\mathrm{C} 2$ field-of-view), the previous ones and the following ones. The apparent speeds and sizes of all these CMEs are then systematically measured in the $\mathrm{C} 2$ (or C3) fields of view using LASCO movies. Among these five CMEs determined from the launch window, the largest ones, in particular the halo CMEs, are particularly considered. Indeed, such perturbations have a higher probability to reach and encounter the Earth's orbit. For a succession of possibly associated CME halo events, the fastest one has been selected as it probably overtakes the previous and slower perturbation. In Table 1, this is the case for the interplanetary event of 1 October 1997 and for the series of events ranging from 30 April 1998 to 4 May 1998. The event of 1 October 1997 will be discussed in more detail below. For the period from 30 April 1998 to 4 May 1998, four successive events were observed in the interplanetary medium and five halo CMEs were observed in the launch window, corresponding to these events. The only ambiguity is in fact for the association of 

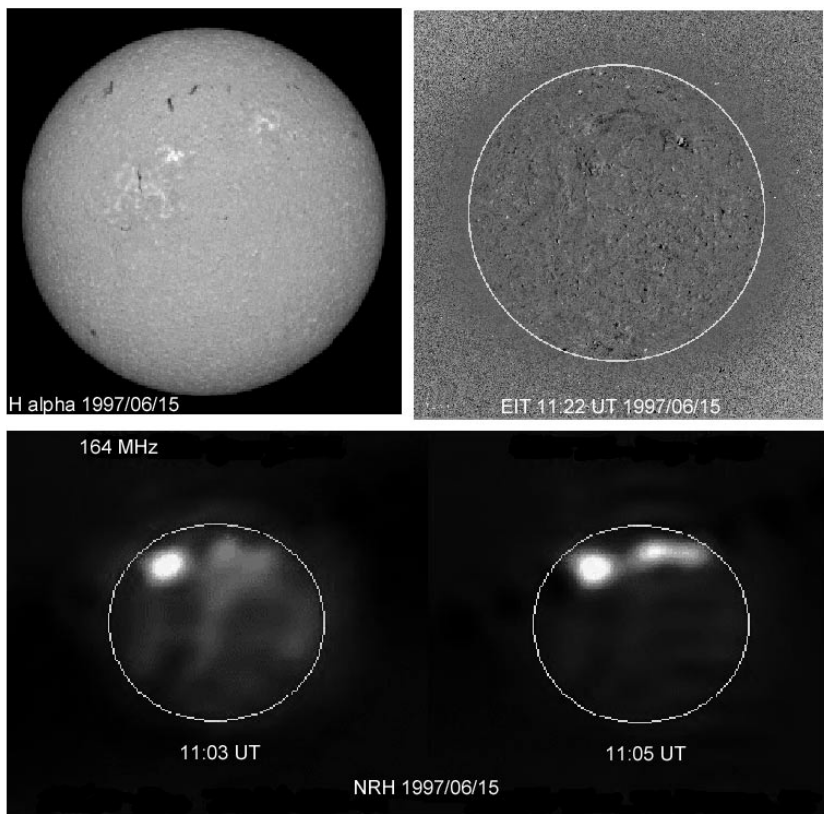

Fig. 1. Top left: $\mathrm{H} \alpha$ observations (BASS 2000 Meudon) on 15 June 1997 showing a filament channel in the Northern Hemisphere close to the disk central meridian. The active region (N17 E02) involved in the flare (see Table 2) is also seen close to the Sun's center. Top right: EIT (195 ̊) difference image showing in black the disappearance of a filament in the North Hemisphere. In white, close to the eastern part of the filament, notice a faint brightening. Bottom: NRH observations at $164 \mathrm{MHz}$ at two successive times, showing first the location of a burst at 11:03 UT (close to the flare start) and the brightening of an extended radio source shaping the EIT filament and probably related to the development on the disk of the CME

the interplanetary ejecta on 4 May 1998 with the CME halos either on 2 May 1998 at 05:31 UT or at 14:06 UT. According to our criteria, the second one (which is fastest) is chosen. Finally, for two cases when the examination of LASCO observations did not allow one to firmly establish an association, EIT (Delaboudinière et al., 1995) and Nançay Radioheliograph (NRH) (Kerdraon and Delouis, 1997), observations have also been analysed (see below). Indeed, as recalled in the Introduction, these observations may provide signatures of the onset of CMEs above the solar disk (see, e.g. Thompson et al., 2000a, b; Delannée et al., 2000; Pohjolainen et al., 2001). For the ICME of 19 June 1997, no CME was reported for several days around 15 June 1997, but combined EIT observations at $195 \AA$ and NRH observations at $164 \mathrm{MHz}$ (Fig. 1) show the development on the disk of an EUV and radio event, with characteristics similar to previously published cases for which LASCO CMEs have been observed: localized radio emission connected to the flare region followed by a large-scale loop-like radio source (see, e.g. Pohjolainen et al., 2001; Maia et al., 2000). For the interplanetary shock of 1 October 1997 , although the closest LASCO CME was a halo, we chose to associate the interplanetary shock to a combination of following CMEs in $\mathrm{C} 2$.

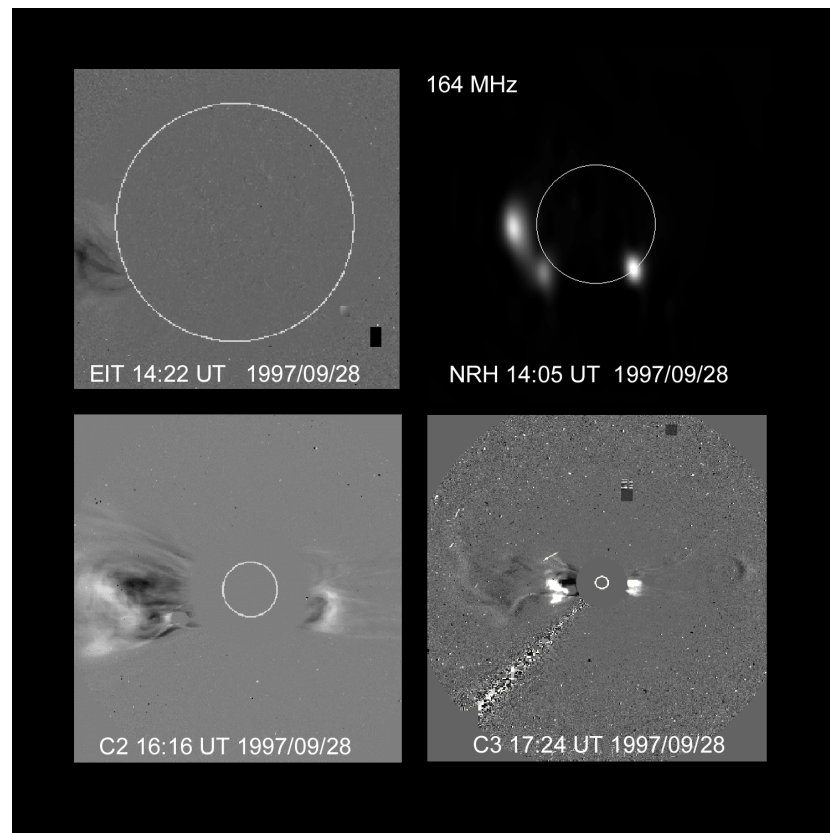

Fig. 2. Top left: EIT (195 A) difference image at 14:22 UT showing the development above the east limb of an eruptive prominence (seen first at 14:13 UT). Top right: NRH observation at $164 \mathrm{MHz}$ showing on the east limb the extended radio loop-like source associated with the CME development (as in, for example, Maia et al., 2000). The source on the southwest is a noise storm present all day long. Bottom: LASCO C2 and C3 difference images showing the development on both hemispheres (at polar angles (PA) $90^{\circ}$ and $265^{\circ}$ ) of CMEs which finally develop a partial halo feature in $\mathrm{C} 3$.

Indeed, they are faster than the halo, appear on both east and west sides in $\mathrm{C} 2$ and later in C3 they form a partial halo. The eastern event is also clearly detected in EIT and with the $\mathrm{NRH}$ at $164 \mathrm{MHz}$ (Fig. 2).

The CMEs selected after all these steps are finally reported in column 7 of Table 1. An association between interplanetary (IP) events and LASCO/CMEs or proxies observed with EIT and NRH is found for 36 of the 40 events listed in Table 1 . In $32 \%$ of the cases, the associated CME corresponds to the CME which was observed the closest in time with $t_{c}$.

Figure 3 displays in light grey the histogram distribution of $\Delta T$ which is the difference between the onset time of the chosen associated CME (column 7) and $t_{c}$ for all the cases of Table 1. The distribution in black is drawn for the 23 cases for which the associated CME (column 7) does not correspond to the CME closest in time with $t_{c}$ (column 6). The distribution in dark grey corresponds to the CMEs of column 6 , i.e. to the CMEs which are the closest in time with $t_{c}$. This last distribution is, as expected, peaked around $\Delta T=0$. Figure 3 shows that a large number of IPs have propagated in the interplanetary medium with a velocity larger than the velocity measured at WIND $(\Delta T \geq 0)$. Even if the associated CME is the one closest to $t_{c}$, there is already an excess of cases with $\Delta T \geq 0$ (dark grey distribution). This excess is largely increased for the distribution of CMEs of column 7 
Table 1. Association between Interplanetary Disturbances (IP) and CMEs

\begin{tabular}{|c|c|c|c|c|c|c|c|}
\hline $\begin{array}{l}\text { Start time of } \\
\text { IP at WIND }\end{array}$ & Type & $\begin{array}{c}\text { Velocity at } 1 \mathrm{AU} \\
V_{\text {WIND }} \mathrm{km} / \mathrm{s}\end{array}$ & $\begin{array}{c}\text { Propagation time } \\
\text { inferred }\end{array}$ & $\begin{array}{c}\text { Launch window } \\
t_{c}\end{array}$ & $\begin{array}{l}\text { LASCO CME } \\
\text { closest to } t_{c}\end{array}$ & $\begin{array}{c}\text { LASCO CME } \\
\text { chosen }\end{array}$ & $\begin{array}{l}K_{p} \\
D_{s t}(\mathrm{nT})\end{array}$ \\
\hline $10-1-97$ & $\mathrm{MC}$ & $440 \pm 30$ & $3 \mathrm{~d} 21 \mathrm{~h} \pm 6 \mathrm{~h}$ & $6-1-97$ & $6-1-97,15: 10$ & $6-1-97,15: 10$ & 6 \\
\hline $00: 22$ & & & & 03:30 $\pm 06: 00$ & Halo & Halo & -73 \\
\hline $9-2-97$ & $\mathrm{MC}$ & $595 \pm 15$ & $2 \mathrm{~d} 21 \mathrm{~h} \pm 2 \mathrm{~h}$ & $6-2-97$ & $7-2-97,00: 30$ & $7-2-97,00: 30$ & 4 \\
\hline $12: 43$ & & & & $15: 30 \pm 02: 00$ & Halo & Halo & -68 \\
\hline $27-2-97$ & $\mathrm{MC}$ & $550 \pm 50$ & $3 \mathrm{~d} 3 \mathrm{~h} \pm 7 \mathrm{~h}$ & $24-2-97$ & $24-2-97,11: 30$ & ambiguous & 7 \\
\hline $17: 30$ & & & & $14: 30 \pm 07: 00$ & $255^{\circ} \mathrm{PA}, 50^{\circ} W_{i}$ & & -86 \\
\hline $10-4-97$ & shock & $475 \pm 25$ & $3 \mathrm{~d} 15 \mathrm{~h} \pm 4 \mathrm{~h}$ & $06-4-97$ & $07-4-97,06: 26$ & $07-4-97,14: 27$ & 6 \\
\hline $12: 57$ & + sheath & & & $22: 00 \pm 04: 00$ & Halo & Halo & -74 \\
\hline $15-5-97$ & $\mathrm{MC}$ & $425 \pm 25$ & $4 \mathrm{~d} \pm 5 \mathrm{~h}$ & $11-5-97$ & $12-5-97,06: 30$ & $12-5-97,06: 30$ & 7 \\
\hline $00: 56$ & & & & 01:00土 05:00 & Halo & Halo & -115 \\
\hline $20-5-97$ & $\mathrm{MC}$ & $340 \pm 10$ & $5 d \pm 3 h$ & $15-5-97$ & $15-5-97,20: 31$ & $16-5-97,12: 45$ & 2 \\
\hline $04: 47$ & & & & 05:00 \pm 03:00 & $270^{\circ} \mathrm{PA}, 36^{\circ} W_{i}$ & $277^{\circ} \mathrm{PA}, 57^{\circ} W_{i}$ & -17 \\
\hline $25-5-97$ & shock & $320 \pm 5$ & $5 \mathrm{~d} 8 \mathrm{~h} \pm 2 \mathrm{~h}$ & $20-05-97$ & $21-05-97,21: 30$ & no associated CME & 2 \\
\hline $13: 23$ & & & & $05: 30 \pm 02: 00$ & $93^{\circ} \mathrm{PA}, 14^{\circ} W_{i}$ & & -13 \\
\hline $26-5-97$ & $\mathrm{MC}$ & $335 \pm 25$ & $5 \mathrm{~d} 2 \mathrm{~h} \pm 9 \mathrm{~h}$ & $21-5-97$ & $21-5-97,04: 19$ & $21-5-97,21: 00$ & 6 \\
\hline 09:10 & & & & 07:00 \pm 09:00 & $260^{\circ} \mathrm{PA}, 30^{\circ} W_{i}$ & $260^{\circ} \mathrm{PA}, 160^{\circ} W_{i}$ & -73 \\
\hline $19-6-97$ & $\mathrm{MC}$ & $390 \pm 5$ & $4 \mathrm{~d} 9 \mathrm{~h} \pm 1 \mathrm{~h}$ & $14-6-97$ & $*$ & 15-6-97, 11:30 & 3 \\
\hline $00: 12$ & & & & 15:00 $\pm 01: 00$ & & EIT and NRH & -28 \\
\hline $15-7-97$ & Flux & $360 \pm 20$ & $4 \mathrm{~d} 17 \mathrm{~h} \pm 6 \mathrm{~h}$ & $10-7-97$ & $11-7-97,01: 30$ & ambiguous & $4-$ \\
\hline 06:00 & Rope & & & 13:00 $\pm 06: 00$ & $85^{\circ} \mathrm{PA}, 67^{\circ} W_{i}$ & & -49 \\
\hline $3-8-97$ & Flux & $500 \pm 5$ & $3 \mathrm{~d} 10 \mathrm{~h} \pm 1 \mathrm{~h}$ & $31-7-97$ & $30-7-97,19: 32$ & $30-7-97,19: 32$ & 5 \\
\hline 14:00 & Rope & & & 04:00 $\pm 01: 00$ & $93^{\circ} \mathrm{PA}, 50^{\circ} W_{i}$ & $93^{\circ} \mathrm{PA}, 50^{\circ} W_{i}$ & -50 \\
\hline $2-9-97$ & shock & $365 \pm 15$ & $4 \mathrm{~d} 16 \mathrm{~h} \pm 5 \mathrm{~h}$ & $29-8-97$ & $29-8-97,05: 29$ & $29-8-97,05: 29$ & 3 \\
\hline \multirow[t]{2}{*}{$22: 38$} & & & & $06: 30 \pm 05: 00$ & $246^{\circ} \mathrm{PA}, 54^{\circ} W_{i}$ & $246^{\circ} \mathrm{PA}, 54^{\circ} W_{i}$ (see text) & 13 \\
\hline & & & & & & EIT & \\
\hline $3-9-97$ & $\mathrm{MC}$ & $415 \pm 5$ & $4 \mathrm{~d} 3 \mathrm{~h} \pm 1 \mathrm{~h}$ & $30-8-97$ & $30-8-97,01: 30$ & $30-8-97,01: 30$ & $6+$ \\
\hline 08:37 & & & & $05: 30 \pm 01: 00$ & Halo & Halo & -98 \\
\hline $18-9-97$ & shock & $360 \pm 20$ & $4 \mathrm{~d} 18 \mathrm{~h} \pm 6 \mathrm{~h}$ & $13-9-97$ & $13-9-97,11: 33$ & ambiguous & 5 \\
\hline $00: 21$ & & & & $06: 30 \pm 6 \mathrm{~h}$ & $95^{\circ} \mathrm{PA}, 70^{\circ} W_{i}$ & & -56 \\
\hline $22-9-97$ & $\mathrm{MC}$ & $475 \pm 15$ & $3 \mathrm{~d} 15 \mathrm{~h} \pm 2 \mathrm{~h}$ & $18-9-97$ & $17-9-97,20: 28$ & $17-9-97,20: 28$ & $4+$ \\
\hline 01:00 & & & & $10: 00 \pm 01: 00$ & Halo & Halo & -36 \\
\hline $01-10-97$ & shock & $420 \pm 20$ & $4 \mathrm{~d} 2 \mathrm{~h} \pm 4 \mathrm{~h}$ & $27-9-97$ & 28-9-97, 01:08 & $28-09-97,14: 28$ & 6 \\
\hline \multirow[t]{3}{*}{$00: 20$} & + sheath & & & $22: 30 \pm 4 \mathrm{~h}$ & Halo & $90^{\circ} \mathrm{PA}, 77^{\circ} W_{i}$ & -98 \\
\hline & & & & & & $28-9-97,15: 31$ & \\
\hline & & & & & & $265^{\circ} \mathrm{PA}, 57^{\circ} W_{i}$ (see text) & \\
\hline $10-10-97$ & shock & $450 \pm 20$ & $3 \mathrm{~d} 19 \mathrm{~h} \pm 4 \mathrm{~h}$ & $06-10-97$ & $06-10-97,07: 55$ & $06-10-97,10: 31$ & 4 \\
\hline $02: 27$ & & & & $07: 30 \pm 04: 00$ & $69^{\circ} \mathrm{PA}, 39^{\circ} W_{i}$ & $267^{\circ} \mathrm{PA}, 73^{\circ} W_{i}$ & -56 \\
\hline $10-10-97$ & $\mathrm{MC}$ & $450 \pm 10$ & $3 \mathrm{~d} 19 \mathrm{~h} \pm 2 \mathrm{~h}$ & $06-10-97$ & $06-10-97,15: 28$ & $06-10-97,15: 28$ & $6+$ \\
\hline $15: 48$ & & & & $21: 00 \pm 02: 00$ & $175^{\circ} \mathrm{PA}, 150^{\circ} W_{i}$ & $175^{\circ} \mathrm{PA}, 150^{\circ} W_{i}$ & -130 \\
\hline $23-10-97$ & shock + & $360 \pm 20$ & $4 \mathrm{~d} 18 \mathrm{~h} \pm 6 \mathrm{~h}$ & $18-10-97$ & $19-10-97,04: 42$ & $19-10-97,04: 42$ & 3 \\
\hline 07:02 & sheath & & & $14: 00 \pm 6 h$ & $90^{\circ} \mathrm{PA}, 77^{\circ} W_{i}$ & $90^{\circ} \mathrm{PA}, 77^{\circ} W_{i}$ & -35 \\
\hline $24-10-97$ & shock & $450 \pm 10$ & $3 \mathrm{~d} 19 \mathrm{~h} \pm 2 \mathrm{~h}$ & $20-10-97$ & $21-10-97,01: 27$ & $21-10-97,01: 27$ & 5 \\
\hline $11: 20$ & + sheath & & & $16: 30 \pm 02: 00$ & $109^{\circ} \mathrm{PA}, 87^{\circ} W_{i}$ & $109^{\circ} \mathrm{PA}, 87^{\circ} W_{i}$ & -60 \\
\hline $06-11-97$ & $\mathrm{MC}$ & $465 \pm 5$ & $3 \mathrm{~d} 16 \mathrm{~h} \pm 1 \mathrm{~h}$ & $03-11-97$ & $03-11-97,05: 28$ & $04-11-97,06: 10$ & 4 \\
\hline $22: 30$ & & & & 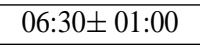 & $229^{\circ} \mathrm{PA}, 90^{\circ} W_{i}$ & Halo & -110 \\
\hline $22-11-97$ & $\mathrm{MC}$ & $495 \pm 25$ & $3 \mathrm{~d} 11 \mathrm{~h} \pm 4 \mathrm{~h}$ & $18-11-97$ & $19-11-97,12: 27$ & $19-11-97,12: 27$ & $7+$ \\
\hline 08:55 & & & & $22: 00 \pm 04: 00$ & Halo & Halo & -108 \\
\hline $10-12-97$ & $\mathrm{MC}$ & $380 \pm 20$ & $4 \mathrm{~d} 11 \mathrm{~h} \pm 5 \mathrm{~h}$ & $05-12-97$ & $05-12-97,08: 27$ & $06-12-97,10: 27$ & 4 \\
\hline $04: 23$ & & & & $17: 30 \pm 05: 00$ & $268^{\circ} \mathrm{PA}, 103^{\circ} W_{i}$ & $304^{\circ} \mathrm{PA}, 250^{\circ} W_{i}$ & -56 \\
\hline $30-12-97$ & $\mathrm{MC}$ & $375 \pm 5$ & $4 \mathrm{~d} 12 \mathrm{~h} \pm 1 \mathrm{~h}$ & $25-12-97$ & $26-12-97,02: 31$ & $26-12-97,02: 31$ & $5-$ \\
\hline 01:00 & & & & 13:00土01:00 & $175^{\circ} \mathrm{PA}, 250^{\circ} W_{i}$ & $175^{\circ} \mathrm{PA}, 250^{\circ} W_{i}$ & -77 \\
\hline $06-01-98$ & $\mathrm{MC}$ & $370 \pm 10$ & $4 \mathrm{~d} 14 \mathrm{~h} \pm 3 \mathrm{~h}$ & $01-01-98$ & 02-01-98, 04:37 & $02-01-98,23: 28$ & $4+$ \\
\hline $13: 19$ & & & & $23: 30 \pm 03: 00$ & $290^{\circ} \mathrm{PA}, 16^{\circ} W_{i}$ & Halo & -77 \\
\hline $24-01-98$ & Ejecta & $425 \pm 15$ & $4 d \pm 3 h$ & $20-01-98$ & $20-01-98,10: 11$ & $21-01-98,06: 37$ & 1 \\
\hline $04: 49$ & & & & 05:00土 03:00 & $43^{\circ} \mathrm{PA}, 23^{\circ} W_{i}$ & Halo & 21 \\
\hline
\end{tabular}


Table 1. continued

\begin{tabular}{|c|c|c|c|c|c|c|c|}
\hline $\begin{array}{l}\text { Start time of } \\
\text { IP at WIND }\end{array}$ & Type & $\begin{array}{c}\text { Velocity at } 1 \mathrm{AU} \\
V_{\text {WIND }} \mathrm{km} / \mathrm{s}\end{array}$ & $\begin{array}{c}\text { Propagation time } \\
\text { inferred }\end{array}$ & $\begin{array}{l}\text { Launch window } \\
t_{c} \\
\end{array}$ & $\begin{array}{l}\text { LASCO CME } \\
\text { closest to } t_{c}\end{array}$ & $\begin{array}{c}\text { LASCO CME } \\
\text { chosen }\end{array}$ & $\begin{array}{l}K_{p} \\
D_{s t}(\mathrm{nT})\end{array}$ \\
\hline $28-01-98$ & shock + & $430 \pm 10$ & $3 \mathrm{~d} 23 \mathrm{~h} \pm 2 \mathrm{~h}$ & $24-01-98$ & $24-01-98,16: 43$ & $25-01-98,15: 26$ & 2 \\
\hline $16: 03$ & sheath & & & $17: 00 \pm 02: 00$ & $290^{\circ} \mathrm{PA}, 20^{\circ} W_{i}$ & Halo & -8 \\
\hline $31-01-98$ & shock + & $410 \pm 10$ & $4 \mathrm{~d} 4 \mathrm{~h} \pm 2 \mathrm{~h}$ & $27-01-98$ & $27-01-98,20: 24$ & $26-01-98,23: 27$ & 4 \\
\hline $15: 57$ & sheath & & & $13: 00 \pm 02: 00$ & $103^{\circ} \mathrm{PA}, 35^{\circ} W_{i}$ & $257^{\circ} \mathrm{PA}, 54^{\circ} W_{i}$ & -26 \\
\hline $03-02-98$ & $\mathrm{MC}$ & $350 \pm 10$ & $4 \mathrm{~d} 31 \mathrm{~h} \pm 3 \mathrm{~h}$ & $30-01-98$ & $30-01-98,14: 31$ & $31-01-98,12: 41$ & 2 \\
\hline 13:09 & & & & $16: 00 \pm 03: 00$ & $272^{\circ} \mathrm{PA}, 21^{\circ} W_{i}$ & $73^{\circ} \mathrm{PA}, 68^{\circ} W_{i}$ & -34 \\
\hline $17-02-98$ & Flux rope & $400 \pm 10$ & $4 \mathrm{~d} 7 \mathrm{~h} \pm 2 \mathrm{~h}$ & $12-02-98$ & $12-02-98,16: 00$ & $12-02-98,14: 27$ & 4 \\
\hline 03:00 & & & & $20: 00 \pm 02: 00$ & $20^{\circ} \mathrm{PA}, 140^{\circ} W_{i}$ & $273^{\circ} \mathrm{PA}, 48^{\circ} W_{i}$ & -100 \\
\hline $18-02-98$ & $\mathrm{MC}$ & $415 \pm 5$ & $4 \mathrm{~d} 3 \pm 01: 00$ & $14-02-98$ & $14-02-98,07: 00$ & $14-02-98,10: 55$ & $5+$ \\
\hline 08:00 & & & & 05:00 \pm 01:00 & $130^{\circ} \mathrm{PA}, 220^{\circ} W_{i}$ & $289^{\circ} \mathrm{PA}, 26^{\circ} W_{i}$ & -51 \\
\hline $04-03-98$ & $\mathrm{MC}$ & $390 \pm 10$ & $4 \mathrm{~d} 8 \mathrm{~h} \pm 2 \mathrm{~h}$ & $28-02-98$ & $28-02-98,10: 22$ & $28-02-98,12: 48$ & 1 \\
\hline 11:00 & & & & 03:00 $\pm 02: 00$ & $253^{\circ} \mathrm{PA}, 54^{\circ} W_{i}$ & $220^{\circ} \mathrm{PA}, 200^{\circ} W_{i}$ & -36 \\
\hline $23-04-98$ & shock + & $430 \pm 30$ & $4 \mathrm{~d} 6 \mathrm{~h} \pm 6 \mathrm{~h}$ & $19-04-98$ & 20-04-98, 07:05 & 20-04-98, 10:07 & 6 \\
\hline $17: 15$ & sheath & & & $17: 00 \pm 6 \mathrm{~h}$ & $84^{\circ} \mathrm{PA}, 98^{\circ} W_{i}$ & $318^{\circ} \mathrm{PA}, 264^{\circ} W_{i}$ & -69 \\
\hline $30-04-98$ & shock & $365 \pm 5$ & $4 \mathrm{~d} 16 \mathrm{~h} \pm 1 \mathrm{~h}$ & $25-04-98$ & $25-04-98,15: 11$ & $27-04-98,08: 56$ & 4 \\
\hline 08:12 & & & & $16: 00 \pm 1 \mathrm{~h}$ & $89^{\circ} \mathrm{PA}, 59^{\circ} W_{i}$ & Halo & 34 \\
\hline $01-05-98$ & $\mathrm{MC}$ & $580 \pm 20$ & $2 \mathrm{~d} 22 \mathrm{~h} \pm 2 \mathrm{~h}$ & $28-04-98$ & $29-04-98,16: 58$ & $29-04-98,16: 58$ & $7-$ \\
\hline $21: 11$ & & & & 23:00 $\pm 02: 00$ & Halo & Halo & -85 \\
\hline $03-05-98$ & shock & $490 \pm 10$ & $3 \mathrm{~d} 12 \mathrm{~h} \pm 2 \mathrm{~h}$ & $30-04-98$ & $29-04-98,16: 58$ & $01-05-98,23: 40$ & 5 \\
\hline $17: 05$ & & & & 05:00 $\pm 02: 00$ & Halo & Halo & -70 \\
\hline $04-05-98$ & Ejecta & $775 \pm 75$ & $2 \mathrm{~d} 5 \mathrm{~h} \pm 5 \mathrm{~h}$ & $01-05-98$ & 01-05-98, 23:40 & 02-05-98, 14:06 & 9 \\
\hline $02: 03$ & & & & $21: 00 \pm 05: 00$ & Halo & Halo & -205 \\
\hline $02-06-98$ & Flux rope & $420 \pm 10$ & $4 \mathrm{~d} 2 \mathrm{~h} \pm 2 \mathrm{~h}$ & $29-05-98$ & 29-05-98,11-27 & 29-05-98,13:31 & 3 \\
\hline $10: 00$ & & & & 08:00土 02:00 & $74^{\circ} \mathrm{PA}, 48^{\circ} W_{i}$ & $358^{\circ} \mathrm{PA}, 83^{\circ} W_{i}$ (see text) & 11 \\
\hline $13-06-98$ & $\mathrm{MC}$ & $380 \pm 5$ & $4 \mathrm{~d} 11 \mathrm{~h} \pm 1 \mathrm{~h}$ & 09-06-98 & 09-06-98, 08:27 & 09-06-98, 09:27 & $4+$ \\
\hline $18: 26$ & & & & $07: 30 \pm 01: 00$ & $101^{\circ} \mathrm{PA}, 51^{\circ} W_{i}$ & $208^{\circ} \mathrm{PA}, 100^{\circ} W_{i}$ & -55 \\
\hline $25-06-98$ & $\mathrm{MC}$ & $450 \pm 20$ & $3 \mathrm{~d} 19 \mathrm{~h} \pm 4 \mathrm{~h}$ & $21-06-98$ & 21-06-98, 18:15 & $22-06-98,07: 34$ & 7 \\
\hline $15: 43$ & & & & $19: 00 \pm 04: 00$ & $265^{\circ} \mathrm{PA}, 50^{\circ} W_{i}$ & $193^{\circ} \mathrm{PA}, 105^{\circ} W_{i}$ & -101 \\
\hline
\end{tabular}

(light grey), which takes into account the additional criteria described above. This shows that, in agreement with former studies (Gopalswamy et al., 2000a; St Cyr et al., 2000), most interplanetary perturbations are partly decelerated in the interplanetary medium, reaching at $1 \mathrm{AU}$ a velocity close to the mean solar wind velocity. This is also consistent with the previous findings that the average transit speed of interplanetary shocks from the Sun is higher than the in situ measurement of the velocity (e.g. Sheeley et al., 1985). The distribution in $\Delta T$ of the chosen CMEs (light grey) is relatively narrow, since $\simeq 75 \%$ of the events lie between $-6 \mathrm{~h}$ and $+18 \mathrm{~h}$. This time must be compared to the transit time between the Sun and the Earth (3 to 4 days). The most extreme cases of our histogram are on one side (between -12 and $-18 \mathrm{~h}$ ) the MC of 22 September 1997 (notice that this extreme case is not an effect of our selection, since the associated CME in column 7 is also the closest one to $t_{c}$ ) and the interplanetary shock of 31 January 1998 and on the other side (greater than $30 \mathrm{~h}$ ), the magnetic cloud of 20 May 1997 and even more extreme the interplanetary shocks of 30 April 1998 and 3 May 1998. It is worthwhile noting that extreme values for $\Delta T$ are already found for the CMEs in column 6.
Figure 4 displays the scatter points of the CME's apparent

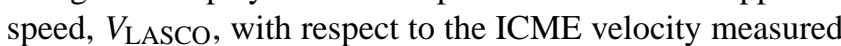
at $1 \mathrm{AU}, V_{\text {WIND. }} V_{\mathrm{LASCO}}$ is derived using the list provided by Yashiro et al. at http://cdaw.gsfc.nasa.gov/CMElist. It is the speed at the last height of measurement in the C3 fieldof-view derived from a quadratic fit or the derived speed at $20 R_{S}$ in the case where the CME is still detected at that height. In agreement with the previous studies of Sheeley et al. (1985) and Webb et al. (2000), no strong relation exists between the projected speeds of CMEs measured by LASCO and the velocity of the disturbances at 1 AU. It must be noticed that the highest velocities measured at $1 \mathrm{AU}$ are not associated with the cases of high projected velocity in the field-of-view of the coronagraphs. This remains true, even if the two CMEs with velocities measured with LASCO above $1500 \mathrm{~km} / \mathrm{s}$, i.e. the CMEs of 20 and 27 April 1998 which are close to the limb events and associated with IP shocks are not considered. Of course, these results are most probably strongly biased by the fact that the speed can be measured only in projection on the sky plane with no indication of the 3-D structure of the CME. There is also no correlation between $\Delta T$ and $V_{\text {LASCO }}$ (Fig. 5), and the highest values of 


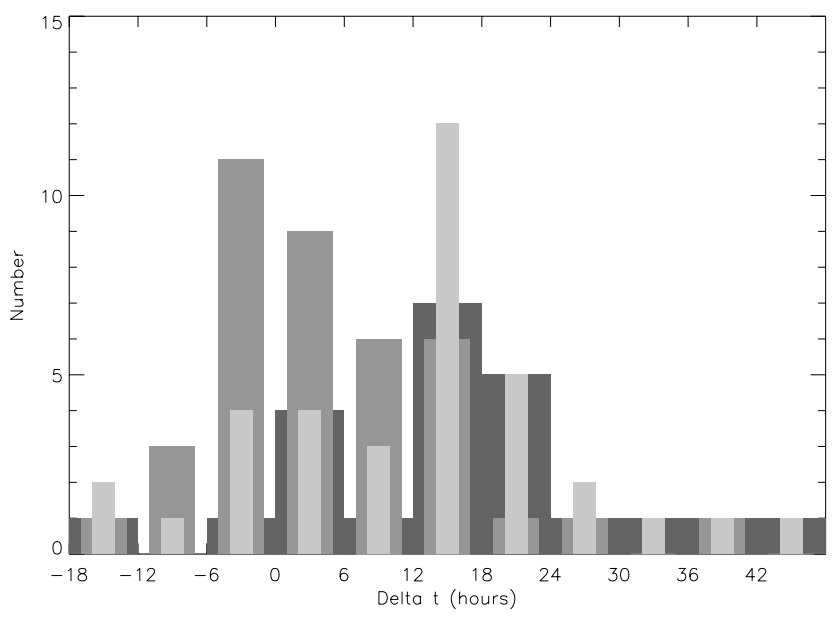

Fig. 3. In light grey: Histogram of $\Delta T$ (difference between the onset time of the chosen CME (column 7, Table 1) and $t_{c}$ (column 5)) for all the cases of Table 1. In black: same histogram for cases when the chosen CME (column 7) is different from the CME in column 6 of Table 1. In dark grey: same histogram for the CMEs of column 6 closest to $t_{c}$.



Fig. 4. Scatter plot of the CME's apparent speed $V_{\text {LASCO }}$ with respect to the Ips velocity $V_{\text {WIND }}$ measured at WIND.

$V_{\text {LASCO }}$ do not correspond to the cases with large $\Delta T$. This is to be expected, since the transit time of the perturbation to $1 \mathrm{AU}$ not only depends on the speed of the perturbation, but also on the state of the interplanetary medium. This is clearly seen when plotting the transit time as a function of $V_{\text {LASCO }}$. The transit times are only loosely correlated with the CME speed measured by LASCO. This is in agreement with the results of Cane et al. (2000).

Figures 6 and 7 display, respectively, in dark and light grey the polar angle (PA) and the apparent heliographic latitude distributions for the CMEs identified in columns 6 and 7 of Table 1. These distributions are compared to similar distributions for all the CMEs identified by LASCO (St Cyr et al., 2000) during the same period (black histograms). The light grey PA distributions of the CMEs associated with interplanetary disturbances are quite different from the general CME

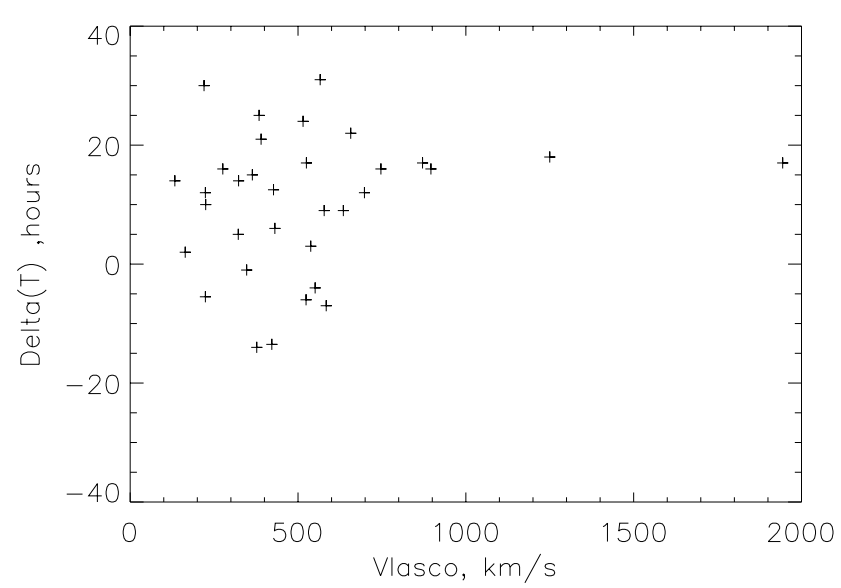

Fig. 5. Scatter plot of $\Delta T$ with respect to the apparent velocity measured in LASCO $V_{\text {LASCO}}$.

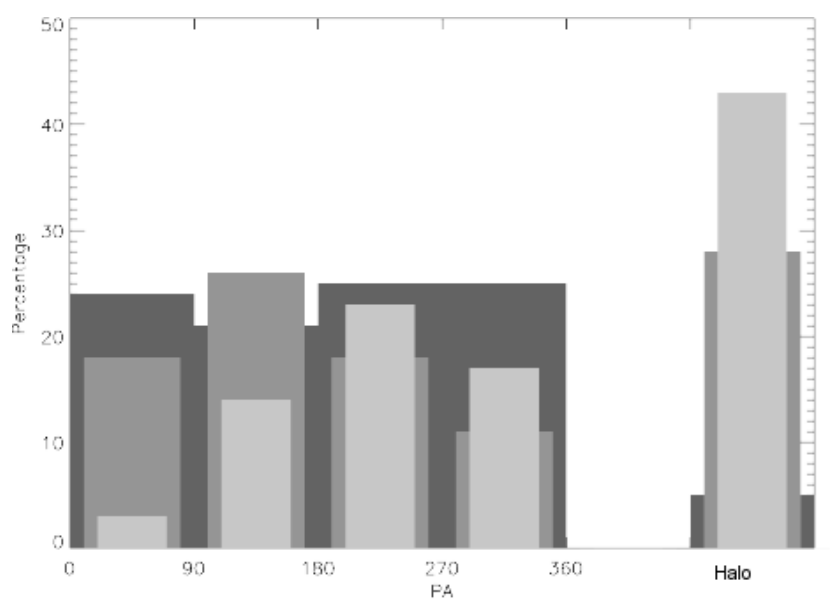

Fig. 6. In light grey: Polar angle (PA) distribution for the CMEs identified in column 7 of Table 1. In dark grey: same histogram for the CMEs identified in column 6 of Table 1. In black: same histogram for all the CMEs identified in LASCO during the same period.

distribution. While the general (black) distribution is rather flat and exhibits a very small proportion of halo events (less than 10\%), the distribution of the selected events (light grey) shows a strong depletion in the 0-90 PA interval (northeast limb) and a strong concentration of halo events (more than $40 \%$ of the selected cases). An increase in the proportion of halo events (around 30\%), compared to the general distribution, is already observed for the CMEs in column 6, i.e. showing that halo events have a higher probability of reaching the Earth than other CMEs. Figure 7 also shows that during the analysed period, the selected CMEs have a slight tendency to be more concentrated in the southern solar hemisphere, and can be seen at high latitudes. This tendency is already seen for the distribution of CMEs from column 6 . Figure 8 shows the distribution of the CME width for our selection (column 7 and light grey histogram), compared to the general distribution (black histogram). While more than $60 \%$ 


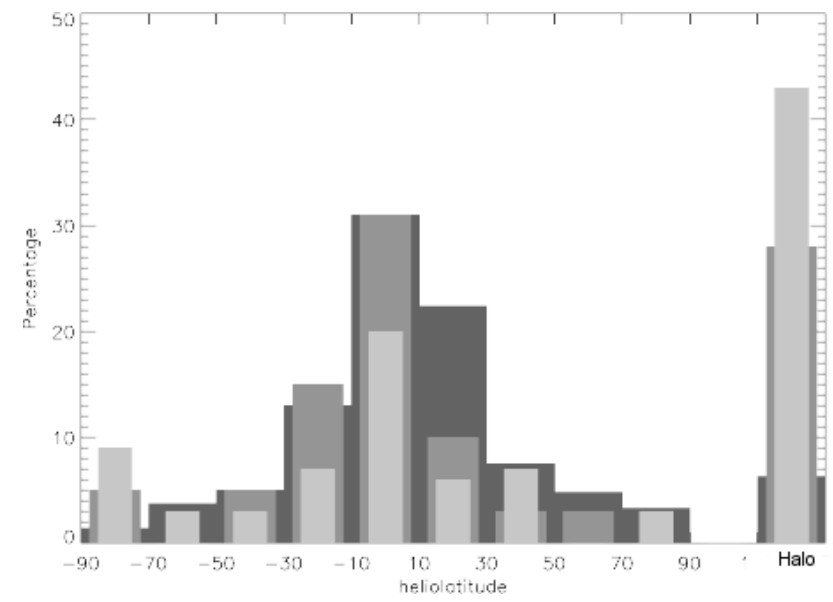

Fig. 7. In light grey: heliolatitude distribution for the CMEs identified in column 7 of Table 1 . In dark grey: same histogram for the CMEs identified in column 6 of Table 1. In black: same histogram for all the CMEs identified in LASCO during the same period.

of the selected CMEs are larger than $120^{\circ}$ or are halos, on the opposite side, $60 \%$ of the events of the general distribution have a width smaller than $60^{\circ}$. The dark grey distribution for the CMEs of column 6 already shows the tendency of a larger proportion of halos and of a width larger than $120^{\circ}$. This is in agreement with former results (Thompson et al., 1999; Webb et al., 2000), where it is found that a threshold of $120^{\circ}$ in width is representative of CMEs associated with ICMEs detected at $1 \mathrm{AU}$.

The properties of CMEs associated with interplanetary perturbations at $1 \mathrm{AU}$ have been described in this section, either for the CMEs closest to the launch window (column 6) or for the CMEs identified in column 7, which, according to our criteria, have a higher probability of being associated with the interplanetary perturbations than the ones in column 6 . Indeed, some of the CMEs in column 6 are very narrow, and some do not even reach the field-of-view of $\mathrm{C} 3$. Therefore, in the following, the CMEs from column 7 will be considered as the ones associated with the interplanetary perturbations.

\subsection{Solar origin of the associated coronal mass ejections}

As recalled in the Introduction, CMEs are associated with various forms of solar activity, either flares or eruptive prominences/disappearing filaments. In this section, we investigate the type of activity that the CMEs identified in column 7 of Table 1 are related. Given the number of cases studied, the results must be considered as indicating tendencies. Furthermore, one must remember that the association with a flare does not preclude that there is also a filament involved, as shown, for example, in Fig. 1. In the following, the association with a filament means that solar activity has its origin either in the disappearance of large filaments not linked to active regions or to filaments in dispersed active regions. The search for the solar origin of CMEs also provides confirma-



Fig. 8. In light grey: width (in ${ }^{\circ}$ ) distribution for the CMEs identified in column 7 of Table 1 . In dark grey: same histogram for the CMEs identified in column 6 of Table 1. In black: same histogram for all the CMEs identified in LASCO during the same period.

tion of the association between IPs and CMEs reported in Table 1.

The solar origin of the CMEs was searched for with the following procedure:

- Search for an optical or GOES X-ray flare in Solar Geophysical Data close to the time of the first appearance in $\mathrm{C} 2$ of the CME. The list of $\mathrm{H} \alpha$ and GOES X-ray flares, as well as the examination of GOES X-ray plots, were used to identify the different kinds of flaring activity.

- SOHO/EIT images in the EUV domain, as well as Nançay Radioheliograph (NRH) images at metric wavelengths (when available), were also used to search for flaring activity in the cases when the association was not obvious from Solar Geophysical Data.

- The magnetic context in which solar activity is observed was systematically examined using optical images in $\mathrm{H} \alpha$, KIV or K3 (from the French Solar Data Base BASS 2000, http://www.mesola.obspm.fr) and soft X-ray images from YOHKOH (Solar Geophysical Data). Flarerelated radio emission in the interplanetary medium close to the Sun (using WIND observations (Bougeret et al., 1995)) was also examined.

- In the absence of an association with a flaring signature, a systematic search of any signature related to the activation or disappearance of filaments or protuberances was performed using Solar Geophysical Data. In such cases, the information on the location of the associated solar event is of course less precise, given the usual large extent of the filaments. The indications of the location on the Sun are the ones reported by Solar Geophysical Data. In case of ambiguity, EIT and NRH observations were also used. 
Table 2. Solar Origin of CMEs and interplanetary events

\begin{tabular}{|c|c|c|}
\hline $\begin{array}{l}\text { LASCO/CME } \\
\text { or proxy }\end{array}$ & $\begin{array}{l}\text { WIND } \\
\text { type }\end{array}$ & $\begin{array}{l}\text { Solar Source } \\
\text { EIT/NRH input }\end{array}$ \\
\hline 6-1-97, $15: 10$ & MC & Filament-Dispersed Region- S23 W03- DSF- 13:01 UT \\
\hline Halo & & \\
\hline $\begin{array}{l}\text { 7-2-97, 00:30 } \\
\text { Halo }\end{array}$ & MC & Filament-Dispersed Region- S20 W32- DSF- <00:30 UT \\
\hline $\begin{array}{l}\text { 7-4-97, } 14: 27 \\
\text { Halo }\end{array}$ & shock + sheath & $\begin{array}{c}\mathrm{H} \alpha \text { 3N flare and GOES C6.8 X-ray flare - AR8027- S30 E19- 13:54 UT } \\
\text { Interplanetary type II/IV } \\
\text { EIT wave and NRH identification }\end{array}$ \\
\hline $\begin{array}{l}\text { 12-5-97, 06:30 } \\
\text { Halo }\end{array}$ & MC & $\begin{array}{c}\mathrm{H} \alpha 1 \mathrm{~F} \text { flare and GOES C1.3 X-ray flare - AR8038- N21 W09 -04:45 UT } \\
\text { Interplanetary Type II/IV }\end{array}$ \\
\hline $16-5-97,12: 45$ & $\mathrm{MC}$ & $\mathrm{H} \alpha$ SF flare and GOES B2.1 X-ray flare - AR8038- N22 W69 <12:51 UT \\
\hline $277^{\circ} \mathrm{PA}, 57^{\circ} W_{i}$ & & NRH identification \\
\hline $\begin{array}{l}21-5-97,21: 00 \\
260^{\circ} \mathrm{PA}, 160^{\circ} W_{i}\end{array}$ & $\mathrm{MC}$ & $\begin{array}{c}\mathrm{H} \alpha \text { SF flare and GOES M1.3 X-ray flare - AR8040- N05 W12<20:08 UT } \\
\text { Interplanetary type II/ IV } \\
\text { EIT wave and dimming }\end{array}$ \\
\hline $\begin{array}{l}\text { 15-6-97, } 11: 30 \\
\quad \text { EIT }\end{array}$ & $\mathrm{MC}$ & $\begin{array}{l}\text { GOES A3 X-ray flare and NRH burst- 11-00 UT } \\
\text { AR 8052 N17 E02 } \\
\text { EIT and NRH identification (see Fig. 1 and text) }\end{array}$ \\
\hline $30-7-97,19: 32$ & Flux Rope & Ambiguous- Possible association with AR8068- S22E90 \\
\hline $\begin{array}{l}93^{\circ} \mathrm{PA}, 50^{\circ} W_{i} \\
29-8-97,05: 29\end{array}$ & shock & $\begin{array}{l}\text { Active region bordered by filaments } \\
\mathrm{H} \alpha \text { SN flare and GOES C4.0 X-ray flare -AR8076 -N30 E26 04:26 UT }\end{array}$ \\
\hline Halo & & EIT identification (flare + large interconnecting loops) \\
\hline $\begin{array}{l}\text { 30-8-97, 01:30 } \\
\text { Halo }\end{array}$ & MC & $\mathrm{H} \alpha$ SF flare and GOES M1.4 X-ray flare - AR8076- N30 E17 29-8-97 22:56 UT \\
\hline $\begin{array}{l}\text { 17-9-97, 20:28 } \\
\text { Halo }\end{array}$ & $\mathrm{MC}$ & $\mathrm{H} \alpha$ SF flare and GOES M1.0 X-ray flare - AR8084- N21 W84 17:45 UT \\
\hline $\begin{array}{l}28-9-97,14: 28 \\
90^{\circ} \mathrm{PA}, 77^{\circ} W_{i} \\
28-9-97,15: 31\end{array}$ & $\begin{array}{l}\text { shock }+ \\
\text { sheath }\end{array}$ & $\begin{array}{l}\text { GOES C1.0 X-ray flare - AR 8090? - 14:11 UT } \\
\text { behind the limb } \\
\text { EIT eruptive prominence east limb 14:13 UT }\end{array}$ \\
\hline $\begin{array}{l}265^{\circ} \mathrm{PA}, 57^{\circ} W_{i} \\
06-10-97,10: 31 \\
267^{\circ} \mathrm{PA}, 73^{\circ} W_{i}\end{array}$ & shock & $\begin{array}{c}\text { NRH identification (see Fig. 2) } \\
\text { Flare-like behind the limb } \\
\text { EIT identification at 10:07 UT ascending large loop at SW limb } \\
\text { NRH identification at SW limb }\end{array}$ \\
\hline 06-10-97, 15:28 & MC & Filament dispersed region- S73 E42- DSF -14:51 UT \\
\hline $\begin{array}{l}175^{\circ} \mathrm{PA}, 150^{\circ} W_{i} \\
19-10-97,04: 42 \\
90^{\circ} \mathrm{PA}, 77^{\circ} W_{i} \\
21-10-97,01: 27 \\
109^{\circ} \mathrm{PA}, 87^{\circ} W_{i}\end{array}$ & $\begin{array}{l}\text { shock }+ \\
\text { sheath } \\
\text { shock }+ \\
\text { sheath }\end{array}$ & $\begin{array}{c}\begin{array}{c}\text { EIT filament } \\
\text { ambiguous }\end{array} \\
\text { EIT starts in NE also in S } \\
\text { DSF S33 E59 from 20-10-97, 23:49 UT }\end{array}$ \\
\hline $\begin{array}{l}\text { 04-11-97, 06:10 } \\
\text { Halo }\end{array}$ & MC & $\begin{array}{c}\text { EIT observation at 01:21 UT: ejecta near and above east limb } \\
\mathrm{H} \alpha \text { 2B flare and GOES X2.1 X-ray flare - AR8100- S14 W33 05:54 UT } \\
\text { Interplanetary type II/IV }\end{array}$ \\
\hline $\begin{array}{l}\text { 19-11-97, 12:27 } \\
\text { Halo }\end{array}$ & $\mathrm{MC}$ & Ambiguous \\
\hline $06-12-97,10: 27$ & $\mathrm{MC}$ & H $\alpha$ SF flare- AR8113- N18 W44 08:53 UT \\
\hline $\begin{array}{c}304^{\circ} \mathrm{PA}, 250^{\circ} W_{i} \\
26-12-97,02: 31\end{array}$ & $\mathrm{MC}$ & $\begin{array}{l}\text { NRH long duration event } \\
\text { associated with flare activity in AR8124-S24 E14 }\end{array}$ \\
\hline $\begin{array}{l}175^{\circ} \mathrm{PA}, 250^{\circ} W_{i} \\
02-01-98,23: 28 \\
\text { Halo }\end{array}$ & $\mathrm{MC}$ & GOES B6.4 X-ray flare- AR 8124- S17 W90- 23:35 UT \\
\hline $\begin{array}{l}\text { 21-01-98, 06:37 } \\
\text { Halo }\end{array}$ & Ejecta & $\begin{array}{c}\text { Filament-dispersed region S57 E19 - DSF 04-00 UT } \\
\text { Interplanetary type II } \\
\text { EIT filament eruption }\end{array}$ \\
\hline $\begin{array}{l}\text { 25-01-98, 15:26 } \\
\text { Halo }\end{array}$ & $\begin{array}{l}\text { shock }+ \\
\text { sheath }\end{array}$ & $\begin{array}{l}\text { H } \alpha \text { SF flare and GOES C1.1 X-ray flare - AR } 8147 \text { - N21 E25 14:29 UT } \\
\text { Interplanetary type II/IV } \\
\text { EIT filament NE ascending loop and NRH identification at 14:46 UT }\end{array}$ \\
\hline
\end{tabular}


Table 2. (continued)

\begin{tabular}{|c|c|c|}
\hline $\begin{array}{l}\text { LASCO/CME } \\
\text { or proxy }\end{array}$ & $\begin{array}{l}\text { WIND } \\
\text { type }\end{array}$ & $\begin{array}{l}\text { Solar Source } \\
\text { EIT/NRH input }\end{array}$ \\
\hline $26-01-98,23: 27$ & shock + & $\mathrm{H} \alpha$ flare SN and GOES C5.4 X-ray flare - AR 8142 - S17 W55 22:19 UT \\
\hline $257^{\circ} \mathrm{PA}, 54^{\circ} W_{i}$ & sheath & EIT flare and ascending loop \\
\hline $31-01-98,12: 41$ & MC & No solar association \\
\hline $75^{\circ} \mathrm{PA}, 70^{\circ} W_{i}$ & & \\
\hline $12-02-98,14: 27$ & Flux Rope & Filament \\
\hline $273^{\circ} \mathrm{PA}, 48^{\circ} W_{i}$ & & S20 W35 \\
\hline & & EIT filament at 13:34 UT, SW \\
\hline $14-02-98,10: 55$ & $\mathrm{MC}$ & $\mathrm{H} \alpha$ SF flare and GOES B8.5 X-ray flare - AR 8156 - S24 E23- 09:32 UT \\
\hline $289^{\circ} \mathrm{PA}, 26^{\circ} W_{i}$ & & EIT signature at 09:54 UT (ascending loop) \\
\hline $28-02-98,12: 48$ & $\mathrm{MC}$ & Filament- S23 W05- AFS - 11:46 UT \\
\hline $220^{\circ} \mathrm{PA}, 200^{\circ} W_{i}$ & & NRH identification \\
\hline 20-04-98, 10:07 & shock + & flare-like behind $\mathrm{W}$ limb \\
\hline $318^{\circ} \mathrm{PA}, 264^{\circ} W_{i}$ & sheath & GOES M1.4 09:38 UT S 23 \\
\hline & & Interplanetary type II/IV \\
\hline & & NRH at 09:43 UT SW limb (see Maia et al, 2000) \\
\hline $\begin{array}{l}\text { 27-04-98, 08:56 } \\
\text { Halo }\end{array}$ & shock & $\begin{array}{l}\mathrm{H} \alpha 2 \mathrm{~B} \text { flare and GOES X1.0 X-ray flare- AR 8210- S16 E50- 08:55 UT } \\
\text { Interplanetary type II/IV }\end{array}$ \\
\hline & & EIT wave brightest in east \\
\hline $\begin{array}{l}\text { 29-04-98, } 16: 58 \\
\text { Halo }\end{array}$ & $\mathrm{MC}$ & $\begin{array}{l}\mathrm{H} \alpha \text { 3B flare and GOES M } 6.8 \text { X-ray flare - AR 8210- S14 E23 -16:06 UT } \\
\text { Interplanetary type II/IV }\end{array}$ \\
\hline $\begin{array}{l}\text { 01-05-98, 23:40 } \\
\text { Halo }\end{array}$ & shock & $\begin{array}{c}\mathrm{H} \alpha 1 \mathrm{~F} \text { and GOES M1.2 X-ray flare- AR 8210- S18 W06- 22:32 UT } \\
\text { EIT ascending loop at } \simeq 22: 41 \mathrm{UT}\end{array}$ \\
\hline $\begin{array}{l}\text { 02-05-98, 14:00 } \\
\text { Halo }\end{array}$ & $\mathrm{MC}$ & $\begin{array}{c}\mathrm{H} \alpha \text { 3B flare and GOES X1.1 X-ray flare - AR 8210- S15 W15 -13:31 UT } \\
\text { Interplanetary type II/IV }\end{array}$ \\
\hline $29-05-98,13: 31$ & Flux rope & DSF N43 W27 \\
\hline $358^{\circ} \mathrm{PA}, 83^{\circ} W_{i}$ & & EIT filament eruption $\leq 12.35 \mathrm{UT}$ \\
\hline 09-06-98, 09:27 & $\mathrm{MC}$ & Large filament- AR 8230/8240- $\geq S 24, \geq$ W44 \\
\hline $208^{\circ} \mathrm{PA}, 100^{\circ} W_{i}$ & & EIT SW activity from 09:29 UT to $10: 00 \mathrm{UT}$ \\
\hline $22-06-98,07: 34$ & MC & Filament system - S51 W24- DSF -01:19 UT \\
\hline $193^{\circ} \mathrm{PA}, 105^{\circ} W_{i}$ & & \\
\hline
\end{tabular}

Table 2 summarizes the results for the solar origin of the CMEs. The first two columns recall the characteristics of the CME or proxy (first appearance in $\mathrm{C} 2$, location and width in $\mathrm{C} 2$ or halo) and the type of associated interplanetary disturbance (MC, flux rope, ejecta, shock). The third column summarizes information on the origin of the selected CMEs. $\mathrm{H} \alpha$ and GOES X-ray information are reported (start time of the $\mathrm{H} \alpha$ flare or X-ray flare in case of no optical flare). Interplanetary type II and type IV bursts observed by WIND (http://lep694.gsfc.nasa.gov/waves/waves.html) in the decametric/kilometric wavelength range are also indicated. A reliable identification of a solar origin is found for $32 \mathrm{CMEs}$ among the 36 listed in Table 2. It is worthwhile noting that the time delay between the flare or the signature of a disappearing filament and the time of appearance of the CME is for most cases of the order of a few hours, which sounds reasonable based on the studies which were performed on the development of CMEs on the solar surface (e.g. Pohjolainen et al., 2001; Maia et al., 2000). Furthermore, there is some consistency between the location (PA) of the CME and of the flaring signature. Indeed, many halos (but not all) are asso- ciated with flaring signatures close to the disk center. For the other CMEs, there is a good consistency in most cases between the solar quadrant where the flaring signature is observed and the PA of the CME. For the CME observed on 30 July 1997, only a probable association with an active region surrounded by filaments could be found, but no specific signature was observed at the time of the CME onset. Although an EIT signature was found in relation to the Halo CME of 19 November 1997, no flaring or filament activity was found. It is worthwhile noting that among the 40 studied cases of interplanetary disturbances, only 11 are associated with reported interplanetary type II/IV activity observed by WIND in the decametric/kilometric range.

For the 32 cases for which a solar origin could be found, 10 cases were associated with large filament and/or filament activity not related to a flaring active region. The two flux ropes observed in the interplanetary medium for which a solar association is found belong to this category and are associated with filaments close to the solar disk center. For 22 cases, a flare-like signature was found. Similarly to Fig. 1, Fig. 9 displays the histogram distribution of $\Delta T$ for the cases associ- 


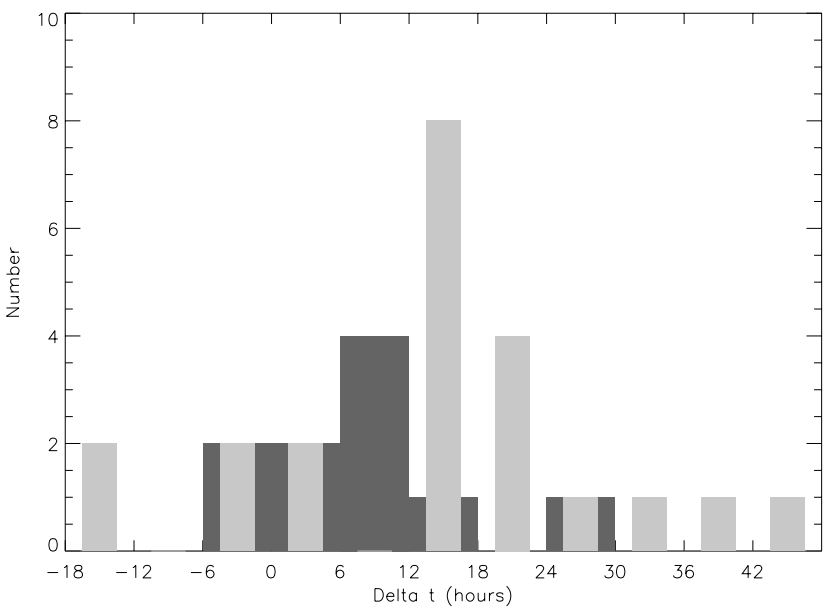

Fig. 9. In grey: Histogram of $\Delta T$ (difference between onset time of chosen CME (column 7 of table 1) and $t_{c}$ (column 5)) for the cases associated with flares (Table 2). In black: same histogram for cases associated with filaments.

ated with filaments (black) and for the cases associated with flares (grey). The histogram for the cases associated with filaments shows a relatively narrow distribution (between -6 and +18 hours), indicating that the associated interplanetary perturbation propagates in most cases close to an almost constant speed in the interplanetary medium, namely the speed measured at $1 \mathrm{AU}$. The most extreme case of this category is the ejecta observed in the interplanetary medium on 24 January 1998 which is associated with an EIT filament, the disapperance of an optical filament and also with an interplanetary type II burst. For this specific case, there is a clear deceleration during the propagation in the interplanetary medium. The interplanetary event of 6 January 1997 is the only other case associated with a filament disappearance observed together with a WIND interplanetary type II burst.

The flare category shows a very different distribution. Most of the events correspond to $\Delta T \geq 0$, indicating a deceleration during the propagation in the interplanetary medium. The most extreme cases discussed in Sect. 2.1 correspond to this category. It is finally worthwhile noting that for the 13 interplanetary shocks (+ shocks and sheaths) reported in Table 1, only one is associated with a disappearing filament starting close to the east limb. All the other cases (9) are associated with flares, 3 of them being behind the limb and 2 of them being at longitudes above $50^{\circ}$. It must be noted that 2 of these shocks associated with either flares behind the limb or at high longitudes had been already reported in Gopalswamy et al. (2001a). For the flare associated cases originating close to the disk center, the lack of detection of the whole feature of the magnetic cloud (in particular, the rotation of the magnetic field) could be explained by the proximity of a sector boundary shortly after the interplanetary disturbance (e.g. interplanetary event of 10 April 1997), or by a succession of flares leading to a succession of IPs (e.g. series of interplanetary events on 2 and 3 September 1997, on 28 and 31 January 1998, on 3 and 4 May 1998).

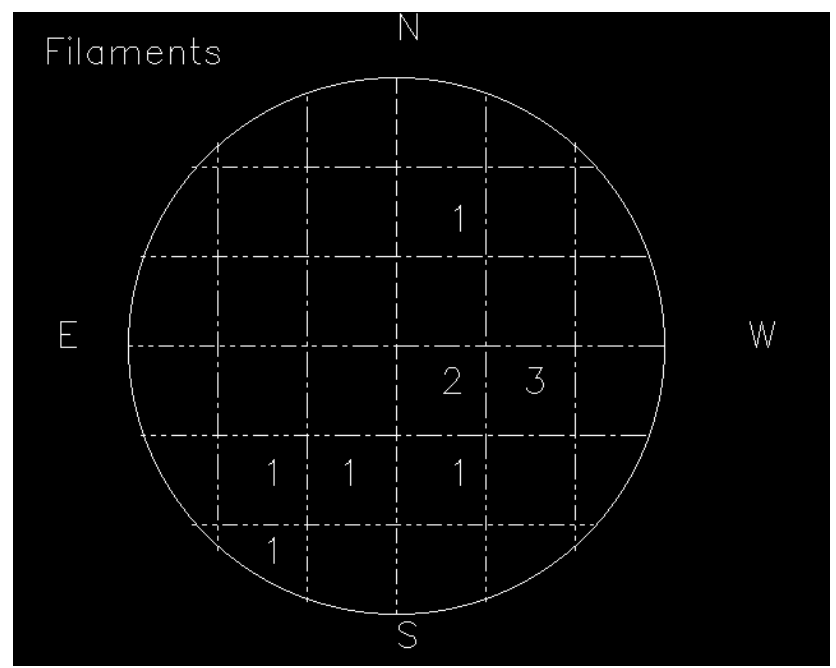

Fig. 10. Positions on the disk of the solar sources of the CMEs selected in Table 1 (column 7) in the case of association with filaments. Lines are drawn every $30^{\circ}$ and numbers of cases in each box are indicated.

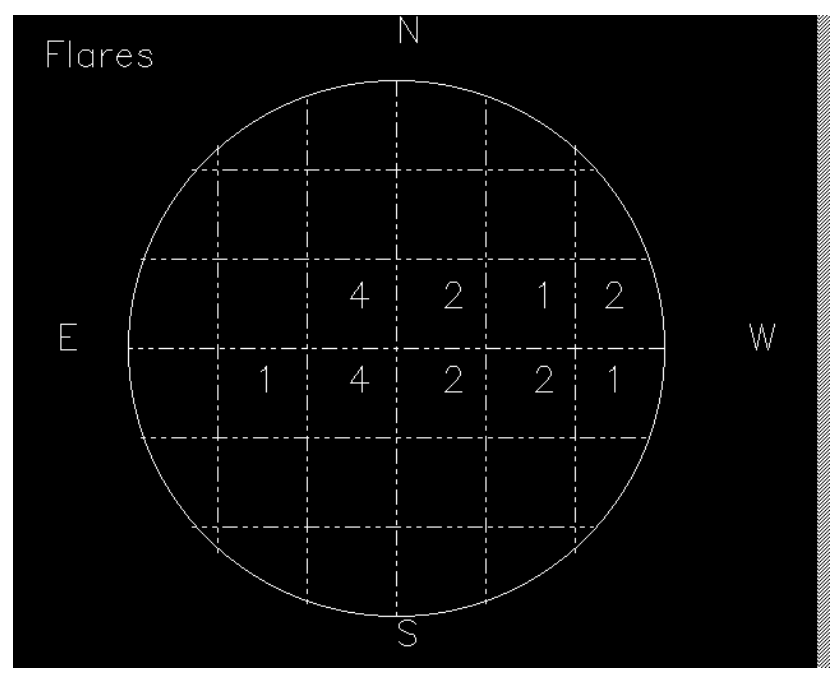

Fig. 11. Positions on the disk of the solar sources of the CMEs chosen in Table 1 (column 7) in the case of association with flares. Lines are drawn every $30^{\circ}$ and numbers of cases in each box are indicated.

Figure 10 shows the position on the solar disk of the filament activity (DSF: Disappearing Solar Filament, AFS: Arch Filament System) for the 10 cases associated with filament disappearance. Most of these events come from the Southern Hemisphere in good consistency with the heliolatitude distribution of Fig. 7. Figure 11 shows the similar distribution for the 19 flare associated cases originating from the solar disk (the three behind the limb events are not reported). As expected for phenomena linked to active regions, the distribution is centered around the solar equator with a slight tendency towards higher western longitudes than eastern. As recalled in the Introduction, for the flare cases, the location 


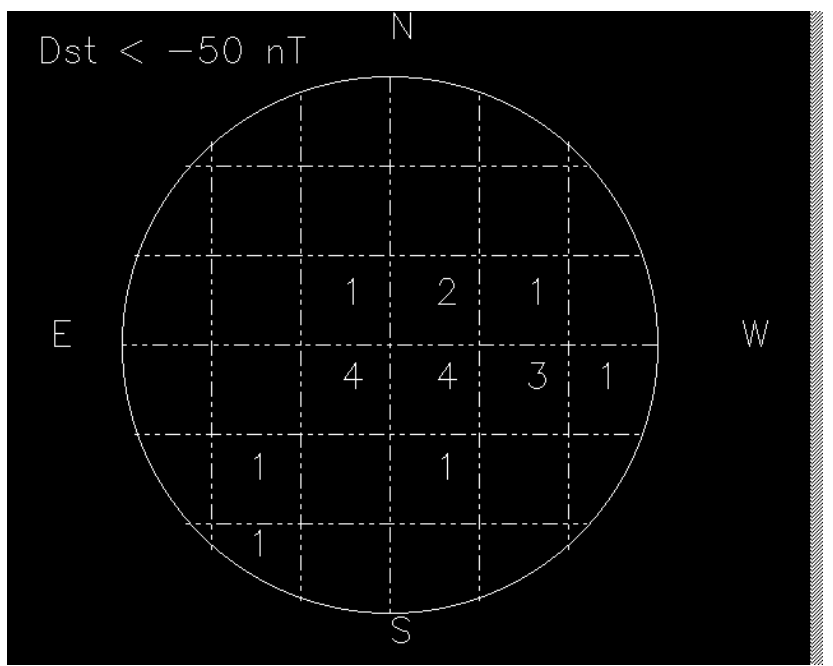

Fig. 12. Positions on the disk of the solar sources of the CMEs chosen in Table 1 (column 7) associated with geomagnetic activity $\left(D_{s t}<-50 \mathrm{nT}\right)$. Lines are drawn every $30^{\circ}$ and numbers of cases in each box are indicated.

of the associated active region does not exactly correspond to the location of the polar angle (PA) of the CME, and the central axes of the CMEs tend to be offset from flaring active regions (Webb, 2000).

Figure 12 shows the positions on the solar disk of the 19 cases which are associated with moderate to strong magnetic storms $\left(D_{s t}<-50 \mathrm{nT}\right)$. A large number of these cases originate from the Southern Hemisphere. Seven are associated with filament disappearance (let us recall that a total of 10 IP events were associated with filament disapperance). The geoeffective filament cases are all located in the Southern Hemisphere and all the sources in Fig. 12 located beyond S30 correspond to filament disappearance. Twelve of the sources in Fig. 12 are flare cases. Compared to the distribution of flare sources in Fig. 11, there may be a small tendency of these geoeffective flares to be located in the Southern Hemisphere (66\% with respect to 53\% for all flares). Although not indicated on the figure, it is worthwhile noting that 3 cases with geomagnetic activity are due to interplanetary shocks associated with behind-the-limb flares.

\section{Discussion}

We summarize below the main results of our study based on 40 interplanetary disturbances selected during the period January 1997-June 1998 and discuss them with respect to results of similar studies published in the literature. The most critical steps concern the establishment of the list of interplanetary perturbations based on specific criteria and the association between these interplanetary perturbations and solar activity. This association is based on a careful examination of many data sets (LASCO C2 and C3, EIT, NRH and $\mathrm{H} \alpha$ images and not on the mere use of CME catalogs and of Solar Geophysical Data reports).
3.1 Selection of interplanetary events observed by WIND in 1997-mid 1998 and association with CMEs

The present study is based on a list of interplanetary events from solar origin that we established through a close examination of the parameters of the solar wind from the WIND observations. The comparison with the list of interplanetary events provided by Gopalswamy et al. (2000a, 2001a) for the same period shows some differences. Among the 40 interplanetary events, 28 are common to both studies. In the present work, the identification and classification of the interplanetary features were performed based on the criteria defined in Sect. 2. The start time of the interplanetary disturbance is the time of the shock which precedes or not the rotation of the magnetic field. This explains the differences between the starting times of the interplanetary disturbances in the present study with respect to the times indicated in Gopalswamy et al. (2000a, 2001a). Apart from 10 cases, an agreement is found between the association reported in Gopalswamy et al. (2000a, 2001a) and the present one.

The association between ICMEs and CMEs performed in this paper on eighteen months of observations contains many more cases than the one performed by Webb et al. (2000) for a period of six months. During the common period, our association is consistent with the one performed by Webb et al. (2000).

\subsection{Interplanetary propagation of solar disturbances}

In the present study, no linear relationship is found between the projected speeds of CMEs measured by LASCO in the field-of-view of the coronagraphs $\mathrm{C} 2$ and $\mathrm{C} 3$ and the velocity of the associated interplanetary disturbances measured at $1 \mathrm{AU}$. No linear relationship is found either between the measured speeds of CMEs and the transit times between the CMEs observed at the Sun and at $1 \mathrm{AU}$. This is in agreement with the results of Webb et al. (2000), St Cyr et al. (2000) and Cane et al. (2000), which state that the measured CME speeds are not correlated with transit speeds, thus pointing out the difficulty of forecasting the arrival at the Earth of these perturbations without a knowledge of the 3-D trajectories or modelling the propagation of the perturbation in the interplanetary medium (Gopalswamy et al., 2000a, 2001c). A large number of the interplanetary perturbations of our sample (in particular, the ones associated with flaring activity at the solar surface) is found to propagate in the interplanetary medium with a velocity larger than the velocity found at $1 \mathrm{AU}(\Delta T>0)$, suggesting that they are decelerated during their propagation in the interplanetary medium. The fact that the IPs that we associated with flaring activity are found to propagate faster in the interplanetary medium than the velocity measured at $1 \mathrm{AU}$ is consistent with the observations of Andrews and Howard (2001) where the type C CME events (see Sect. 1) that they associate with X-ray flares are faster (in the corona) than the type A event they rather associate that with prominence eruptions or filament disappearance. 


\subsection{Solar origin of interplanetary events}

A strong concentration of halo CMEs and of CMEs larger than $120^{\circ}$ is found in this study for the CMEs associated with solar wind perturbations at $1 \mathrm{AU}$. This is in good agreement with the results of many previous analysis (e.g. Webb et al., 2000; Gopalswamy et al., 2000a). A strong depletion of CMEs is also found in the 0-90 PA interval (northeast limb).

\subsection{Solar sources of activity related to CMEs}

In the present study, a reliable identification of a phenomenon on the solar surface is found for 32 of the 36 CMEs of Table 2. In most cases (69\%), the solar source of activity is related to a flaring signature in an active region. This does not of course preclude that large-scale filaments or magnetic structures are involved in the CME development (see, e.g. Fig. 1 and Pohjolainen et al., 2001). This result is somewhat different to the one of Bothmer and Rust (1997), who found for 27 cases among 39 interplanetary events an association between interplanetary magnetic clouds and filament disappearance. This association was, however, based on a study performed on catalogs of DSFs without investigating in detail solar data and without searching for other kinds of solar activity.

For the six months in common, agreement is found between the present study and the one of Webb et al. (2000) concerning the solar source of activity related to a CME. Agreement is also found for 17 associations among $28 \mathrm{com}$ mon cases between the present study and the one of Gopalswamy et al. (2001a).

When looking at the solar sources of activity, it is found that most cases associated with filaments come from the southern part of the solar disk. This is probably related to the fact that most of the important solar activity in early 1997 and in 1998 occurred in the Sun's Southern Hemisphere (Mac Intosh, private communication, see also Webb et al., 2000 and http://sidc.oma.be). The two flux ropes for which a solar origin was found are associated with filaments close to the solar disk center. For the cases associated with flares, the southern tendency is less pronounced. As shown in detailed studies of individual events (e.g. Pohjolainen et al., 2001), even if flare associated, the development of a CME may involve the interaction of magnetic structures at all scales (in the active region, but also between active regions). The slight tendency of higher western longitudes for the origin of the perturbations reaching the Earth probably reflects some convection effects by the solar wind of the interplanetary perturbations during their propagation. The shocks without clouds are found to be mostly associated with either flares behind the limb or with flares occuring in a series of events. In the first case, the detection of the shock without the detection of the cloud could be due to the larger spatial extent of the shock, as already suggested in Gopalswamy et al. (2001a). In the second case, the succession of events in the interplanetary medium could prevent the detection of the development of individual events.
3.5 Association of ICMEs and shocks with interplanetary type II emisions

Only eleven of the interplanetary perturbations listed in the present study are associated with interplanetary type II bursts reported by WIND in the decametric/kilometric wavelength range. This is consistent with the results of Gopalswamy et al. (2001b), where they found that $60 \%$ of fast CMEs were not associated with type II bursts in the decametric range, conversely to the general association found between interplanetary type II bursts and CMEs (Gopalswamy et al., 2000b).

\section{Conclusions}

We recall below the main results of the present study based on 40 interplanetary events (IPs) and on the identification of related CMEs and associated solar activity:

1. An association with LASCO/CME or EIT or NRH radio proxies was found for 36 interplanetary events of the present study.

2. In agreement with previous findings (e.g. Webb et al., 2000; Gopalswamy et al., 2000a), halo CMEs and CMEs larger than $120^{\circ}$ are preferentially associated with solar wind perturbations at $1 \mathrm{AU}$.

3. No linear relationship is found between the projected speeds of CMEs measured by LASCO and the velocity of IPs at $1 \mathrm{AU}$, i.e. no linear relationship between the speeds which can be measured by coronagraphs aboard LASCO and the travel time of the associated perturbation between the Sun and the Earth. This is of course partly due to projection effects and to a lack of knowledge of the 3-D travel geometry of the CME.

4. A reliable identification of a phenomenon on the solar surface was found for 32 of these 36 cases. A large proportion was associated with a flaring signature in an active region. Only 10 cases could not be associated with an active region flaring signature and were found to be associated with the ejection of a large filament and/or with filament activity not related to an active region. The two flux ropes for which a solar origin is found are associated with large-scale filament activity close to the disk center. The interplanetary shocks without magnetic clouds are found mostly in association with flares, either behind the limb or in a series.

5. A large number of the interplanetary perturbations (IPs) associated with the CMEs have been found to propagate in the interplanetary medium with a velocity larger than the velocity measured at $1 \mathrm{AU}$ at $\operatorname{WIND}(\Delta T>0)$. Thus, they have been partly decelerated in the interplanetary medium, reaching at $1 \mathrm{AU}$ a velocity close to the mean solar wind velocity (around $450 \mathrm{~km} / \mathrm{s}$ ). When looking at the associated signature on the solar disk, it 
is found that while there is generally a deceleration for flare events $(\Delta T>0)$, for the cases associated with the ejection of large-scale filaments, the interplanetary perturbation is more likely to propagate at an almost constant speed, i.e. the speed measured at $1 \mathrm{AU}$.

6. A stong concentration of halo CMEs and of CMEs coming from the Southern Hemisphere is observed for the events corresponding to solar wind perturbations at 1 AU in 1997-mid 1998. It is found that most of the cases associated with filaments originate from the southern part of the solar disk. Although less important, this tendency is still present for the cases associated with flares.

7. Among the 40 IP cases, only 11 of them are associated with interplanetary type II activity reported by WIND in the decametric/kilometric wavelength range.

8. The distribution of the solar sources of geo-effective IPs (i.e. associated with moderate to strong magnetic storms: $D_{s t}<-50 \mathrm{nT}$ ) is not strongly different to the general distribution found for the solar sources of IPs. However, a slightly larger proportion of cases associated with large-scale filament activity or disappearance is found with respect to the general list of IPs. Moreover, a large proportion of geo-effective IPs has a solar source in the solar Southern Hemisphere. This may simply reflect the fact that solar activity preferentially occurred in the Southern Hemisphere during that period. A few geo-effective cases are also found in association with shocks without clouds originating from behind-the-limb flares.

The main difficulty for studying the relationship between solar, interplanetary (and geomagnetic phenomena) is primarily linked to our ignorance of the 3-D speed and development in space of coronal mass ejections. Many new results are expected on this topic in the future using in particular the observations of the different experiments aboard the multi-spacecraft STEREO mission. They will provide indeed simultaneous stereoscopic observations of coronal mass ejections (allowing 3-D reconstruction of the propagation of CMEs in the corona), and measurements of the interplanetary perturbations at several locations in the interplanetary medium, thus providing information on the 3-D structure of the interplanetary coronal mass ejections and additional information on the propagation of these magnetic clouds from the Sun to the Earth.

Acknowledgements. We aknowlegde the use of the CME catalog at http://cdaw.gsfc.nasa.gov/CMElist. This CME catalog is generated and maintained by the Center of Solar Physics and Space Weather, the Catholic University of America in cooperation with the Naval Research Laboratory and NASA. SOHO is a project of international cooperation between ESA and NASA. SOHO/EIT was built by an international consortium involving ESA and NASA under the supervision of J. P. Delaboudinière and SOHO/LASCO was built by the Naval Research Laboratory (US), the Max Planck-Institut für Aeronomie (Germany), the Laboratoire d'Astronomie Spatiale
(France), and the University of Birmingham School of Physics and Astronomy (UK). The Nançay Radioheliograph (NRH) is funded by the French Ministry of education, the CNRS and the Région Centre.

The authors are very grateful to the referees for their valuable and constructive comments on the manuscript.

Topical Editor E. Antonucci thanks two referees for their help in evaluating this paper.

\section{References}

Andrews, M. D. and Howard, R. A.: A two type classification of LASCO coronal mass ejection, Space Science Reviews, 95, 1, 147-163, 2001.

Ballatore, P., Villain, J. P., Vilmer, N., and Pick, M.: The influence of the interplanetary medium on SuperDARN radar scattering occurrence, Ann. Geophysicae, 18, 12, 1576-1583, 2001.

Bastian, T. S., Pick, M., Kerdraon, A., Maia, D., and Vourlidas, A.: The Coronal Mass Ejection of 20 April 1998: Direct Imaging at Radio Wavelengths, ApJ, 558, L65-L69, 2001.

Bothmer, V. and Rust, D.: The field configuration of magnetic clouds and the solar cycle, in Coronal Mass Ejections, (Eds) Crooker, N., Joselyn, J. A., and Feynman, J., Geophys. Monograph, AGU, Washington DC, 99, 139-146, 1997.

Bothmer, V. and Schwenn, R.: Eruptive prominences as sources of magnetic cloud in the solar wind, Space Science Reviews, 70, 215-220, 1994.

Bothmer, V. and Schwenn R.: The structure and origin of magnetic clouds in the solar wind, Ann. Geophysicae, 16, 1-24, 1997.

Bougeret, J. L., Kaiser, M. L., Kellog, P. J., Manning, R., Goetz, K., Monson, S. J., Monge, N., Friel, L., Meetre, C. A., Perche, C., Sitruk, L., and Hoang, S.: WAVES: the radio and plasma investigation on the WIND spacecraft, Space Sci. Rev., 71, 231263, 1995.

Brueckner, G. E., Howard, R. A., Koomen, M. J., Korendyke, C. M., Michels, D. J., Moses, J. D., Socker, D. G., Dere, K. P., Lamy, P L., Llebaria, A., Bout, M. V., Schwenn, R., Simnett, G. M., Bedford, D. K., and Eyles, C. J.: The Large Angle Spectrographic Coronagraph (LASCO), Solar Phys., 162, 357-402, 1995.

Burlaga, L.: Terminology for Ejecta in the Solar Wind, EOS Transactions, 82, 39, 433-434, 2001.

Burlaga, L., Sittler, E., Mariani, F., and Schwenn, R.: Magnetic loop behind and interplanetary shock: Voyager, Helios and IMP8 observations, J. Geophys. Res., 86, 6673-6684, 1981.

Burlaga, L. F., Klein, L., Sheeley, N. R., Jr., Michels, D. J., Howard, R. A., Koomen, M. J., Schwenn, R., and Rosenbauer, H.: A magnetic cloud and a coronal mass ejection, Geophys. Res. Letts., 9 , 1317-1320, 1982

Cane, H. V., Richardson, I. G., and St. Cyr, O. C.: Coronal mass ejections, interplanetary ejecta and geomagnetic storms, Geophys. Res. Letts., 27, 21, 3591-3594, 2000.

Cliver, E. W.: Forum: The last word, EOS Transactions, 82, 39, 433-433, 2001.

Delaboudinière, J. P., Artzner, G. E., Brunaud, J., Gabriel, A. H., Hochedez, J. F., Millier, F., Song, X. Y., Au, B., Dere, K. P., Howard, R. A., Kreplin, R., Michels, D. J., Moses, J. D., Defise, J. M., Jamar, C., Rochus, P., Chauvineau, J. P., Marioge, J. P., Catura, R. C., Lemen, J. R., Shing, L., Stern, R. A., Gurman, J. B., Neupert, W. M., Maucherat, A., Clette, F., Cugnon, P., van Dessel, E. L.: EIT: Extreme-ultraviolet Imaging Telescope for the SOHO mission, Solar Phys., 162, 291-312, 1995. 
Delannée, C., Delaboudinière, J. P., and Lamy, P.: Observations of the origin of CMEs in the low corona, Astron. Astrophys., 355, 725-742, 2000.

Gilbert, H. G., Holzer, T. E., Burkepile, J. T., and Hundausen, A.: Active and eruptive prominences and their relationship to Coronal Mass Ejections, ApJ, 537, 503-516, 2000.

Gopalswamy, N., Lara, A., Lepping, R. P., Kaiser, M. L., Berdichevsky, D., and St. Cyr, O. C.: Interplanetary acceleration of Coronal Mass Ejections, Geophys. Res. Letts., 27, 2, 145-148, 2000a.

Gopalswamy, N., Kaiser, M. L., Thompson, B. J., Burlaga, L., Szabo, A., Lara, A., Vourlidas, A., Yashiro, S., and Bougeret, J. L.: Radio-rich solar eruptive events, Geophys. Res. Let., 27, 14271430, 2000b.

Gopalswamy, N., Lara, A., Kaiser, M. L., and Bougeret, J. L.: NearSun and near Earth manifestations of solar eruptions, J. Geophys. Res., 106, 25261-25277, 2001a.

Gopalswamy, N., Yashiro, S., Kaiser, M. L., Howard R. A., and Bougeret, J. L.: Characteristics of Coronal Mass Ejections associated with long wavelength type II radio bursts, J. Geophys. Res., 106, 29219-29 230, 2001b.

Gopalswamy, N., Lara, A., Yashiro, S., Kaiser, M. L., and Howard, R. A.: Predicting the 1 AU Arrival Times of Coronal Mass Ejections, J. Geophys. Res., 106, 29 207-29218, 2001c.

Gosling, J. T.: Corotating and Transient Solar Wind Flows in Three Dimensions, ARA\&A, 34, 35-74, 1996.

Gosling, J. T.: Coronal Mass Ejections: An overview, in: Coronal Mass Ejections, (Eds) Crooker, N., Joselyn, J. A., and Feynman, J., Geophys. Monograph, AGU, Washington DC, 99, 9-16, 1997.

Gosling, J. T., Hildner, E., Mac Queen, R. M., Munro, R. M., Poland, A. L., and Ross, C. L.: Mass ejections from the Sun: a view from Skylab, J. Geophys. Res., 79, 4581-4587, 1974.

Gosling, J. T., Hildner, E., Mac Queen, R. M., Munro, R. M, Poland, A. L., and Ross, C. L.: The speeds of coronal mass ejection events, Solar Phys., 48, 389-397, 1976.

Harrison, R. A.: The nature of solar flares associated with coronal mass ejections, Astron. Astrophys., 304, 585-594, 1995

Kerdraon, A. and Delouis, J.: The Nancay radioheliograph, in: Coronal Physics from radio and space observations, (Ed) Trottet, G., New York, Springer, LNP, 483, 192-201, 1997.

Klein, L. W. and Burlaga, L. F.: Interplanetary magnetic clouds at 1 AU, J. Geophys. Res., 87, 613-624, 1982.

Lindsay, G. M., Luhman, J. G., Russel, C. T., and Gosling, J. T.: Relationships between coronal mass ejection speeds from coronograph images and interplanetary characteristics of associated interplanetary coronal mass ejections, J. Geophys. Res., 104, 12 515-12 524, 1999.

MacQueen, R. M. and Fisher, R. R.: The kinematics of solar inner coronal transients, Solar Phys., 89, 89-102, 1983.

Maia, D., Pick, M., Vourlidas, A., and Howard, R.: Development of Coronal Mass Ejections: Radio Shock Signatures, ApJ, 528, 1, L48-L51, 2000.

Marubashi, K.: Interplanetary Magnetic flux ropes and solar filaments, in: Coronal Mass Ejections, (Eds) Crooker, N., Joselyn, J. A., and Feynmann, J., Washington, AGU, 147-156, 1997.

Munro, R. M., Gosling, J. T., Hildner, E., Mac Queen, R. M., Poland, A. L., and Ross, C. L.: The association of coronal mass ejection transients with other forms of activity, Solar Phys., 61, 201-215, 1979 .

Ogilvie, K. W., Chornay, D. J., Fritzenreiter, R. J., Hunsaker, F., Keller, J., Lobell, J., Miller, G., Scudder, J. D., Sittler, E. C., Jr., Torbert, R. B., Bodet, D., Needell, G., Lazarus, A. J., Stein- berg, J. T., Tappan, J. H., Mavretic, A., and Gergin, E.: SWE, A Comprehensive Plasma Instrument for the Wind Spacecraft, Space Science Reviews, 71, 55-77, 1995.

Pohjolainen, S., Maia, D., Pick, M., Vilmer, N., Khan, J. I., Otruba, W., Warmuth A., Benz, A., Alissandrakis, C., and Thompson, B. J.: On-the-disk development of the Halo Coronal Mass Ejections on 2 May 1998, ApJ, 556, 421-431, 2001.

Russell, C. T.: In Defense of the Term ICME, EOS Transactions, 82, 39, 434-434, 2001.

Rust, D. M.: Spawning and shedding helical magnetic fields in the solar atmosphere, Geophys. Res. Letts., 21, 241-244, 1994.

St Cyr, O. C., and Webb, D. F.: Activity associated with coronal mass ejections at solar minimum: SMM observations from 1984-1986, Solar Phys., 136, 379-394, 1991.

St Cyr, O. C., Howard, R. A., Sheeley, N. R., Jr., Plunkett, S. P., Michels, D. J., Paswaters, S. E., Koomen, M. J., Simnett, G. M., Thompson, B. J., Gurman, J. B., Schwenn, R., Webb, D. F., Hildner, E., and Lamy, P. L.: Properties of Coronal Mass Ejections: SOHO LASCO observations from January 1996 to June 1998, J. Geophys. Res., 105, 18 169-18 186, 2000.

Schwenn, R.: Direct correlations between coronal transients and interplanetary disturbances, Space Science Reviews, 34, 85-99, 1983.

Sheeley, Jr., N. R., Bohlin, J. D., Brueckner, G. E., Purcell, J. D., Scherrer, V. E., Tousey, R., Smith, J. B., Jr., Speich, D. M., Tandberg-Hanssen, E., and Wilson, R. M.: Coronal changes associated with a disappearing filament, Solar Phys., 45, 377-392, 1975

Sheeley, N. R., Howard, R. A., Koomen, M. J., and Michels, D. J.: Association between coronal mass ejections and soft $\mathrm{x}$-ray events, ApJ, 272, 349-354, 1983.

Sheeley, N. R., Howard, R. A., Koomen, M. J., Michels, D. J., Schwenn, R., Mühlauser, K. H., and Rosenbauer, H.: Coronal Mass Ejections and Interplanetary Shocks, J. Geophys. Res., 90, 163-175, 1985.

Srivastava, N., Schwenn, R., Inhester, B., Martin, S. F., and Hanaoka, Y.: Factors related to the Origin of a Gradual Coronal Mass Ejection Associated with an Eruptive Prominence on 21-22 June 1998, ApJ, 534, 468-481, 2000.

Sterling, A. and Hudson, H. S.: YOHKOH SXT Observations of XRay "Dimming" Associated with a Halo Coronal Mass Ejection, ApJ, 491, L55-L58, 1997.

Thompson, B. J., Plunkett, S. P., Gurman, J. B., Newmark, J. S., St. Cyr, O. C., and Michels, D. J.: SOHO/EIT observations of an Earth-directed coronal mass ejection on 12 May 1997, Geophys. Res. Letts., 25, 14, 2461-2464, 1998.

Thompson, B. J., Gurman, J. B., Neupert, W. M., Newmark, J. S., Delaboudinière, J. P., St. Cyr, O. C., Stezelberger, S., Dere, K. P., Howard, R. A., and Michels, D. J.: SOHO/EIT Observations of the 7 April 1997 Coronal Transient: Possible Evidence of Coronal Moreton Waves, ApJ, 517, L151-L154, 1999.

Thompson, B. J., Cliver E. W., Nitta, N., Delannée, C., and Delaboudinière, J. P.: Coronal dimmings and energetic CMEs in April-May 1998, Geophys. Res. Letts., 27, 10, 1431-1434, 2000a.

Thompson, B. J., Reynolds, B., Aurass, H., Gopalswamy, N., Gurman, J. B., Hudson, H. S., Martin, S. F., and St. Cyr, O. C.: Observations of the 24 September 1997 Coronal Flare Waves, Solar Physics, 193, 161-180, 2000b.

Webb, D. F. and Hundhausen, A.: Activity associated with the solar origin of Coronal Mass Ejections, Solar Phys., 108, 383-401, 1987. 
Webb, D. F.: The solar source of Coronal Mass Ejections, in: Eruptive Solar flares, (Eds) Svestka, Z, Jackson, B. V., and Machado, M. E., Springer, New York, 234-247, 1992.

Webb, D. F.: Understanding CMES and their source regions, J. Atmos. Solar-Terr. Phys., 62, 1415-1416, 2000.

Webb, D. F., Cliver, E. W., Crooker, N. U., St Cyr, O. C., and Thompson, B. J.: Relationship of halo coronal mass ejections, magnetic clouds, and magnetic storms, J. Geophys. Res., 105, 27 251-27 260, 2000.

Wilson, R. M. and Hildner, E.: On the association of magnetic clouds with disappearing filaments, J. Geophys. Res., 91, 58675872, 1986.

Zarro, D. M., Sterling, A. C., Thompson, B. J., Hudson, H. S., and Nitta, N.: SOHO-EIT Observations of Extreme-Ultraviolet "Dimming" Associated with a Halo Coronal Mass Ejection, ApJ, 520, L139-L142, 1999.

Zhang, J., Dere, K. P., Howard, R. A., Kundu, M. R., and White, S. W.: On the temporal relationship between Coronal Mass Ejections and Flares, ApJ, 559, 452-552, 2001. 